\title{
Genome-Wide Characterization and Expression Analysis of Aquaporins in Salt cress (Eutrema salsugineum)
}

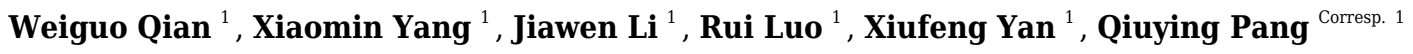 \\ ${ }^{1}$ Alkali Soil Natural Environmental Science Center, Northeast Forestry University/Key Laboratory of Saline-alkali Vegetation Ecology Restoration in Oil \\ Field, Ministry of Education, Harbin, China \\ Corresponding Author: Qiuying Pang \\ Email address: qiuying@nefu.edu.cn
}

Aquaporins (AQPs) serve as water channel proteins and belong to major intrinsic proteins (MIPs) family, functioning in rapidly and selectively transporting water and other small solutes across biological membranes. Importantly, AQPs have been shown to play a critical role in abiotic stress response pathways of plants. As a species closely related to Arabidopsis thaliana, Eutrema salsugineum has been proposed as a model for studying salt resistance in plants. Here we surveyed 35 full-length $A Q P$ genes in $E$. salsugineum, which could be grouped into four subfamilies including 12 plasma membrane intrinsic proteins (PIPs), 11 tonoplast intrinsic proteins (TIPs), 9 NOD-like intrinsic proteins (NIPs), and 3 small basic intrinsic proteins (SIPs) by phylogenetic analysis. EsAQPs were comprised of 237-323 amino acids, with a theoretical molecular weight (MW) of 24.31-31.80 kDa and an isoelectric point ( $\mathrm{pl}$ ) value of 4.73-10.49. Functional prediction based on the NPA motif, aromatic/arginine (ar/R) selectivity filter, Froger's position and specificity-determining position suggested quite differences in substrate specificities of ESAQPs. EsAQPs exhibited global expressions in all organs as shown by gene expression profiles and should be play important roles in response to salt, cold and drought stresses. This study provides comprehensive bioinformation on AQPs in E. salsugineum, which would be helpful for gene function analysis for further studies. 


\section{Genome-Wide Characterization and Expression}

2 Analysis of Aquaporins in Salt cress (Eutrema

3 salsugineum)

4

5

6

7

8

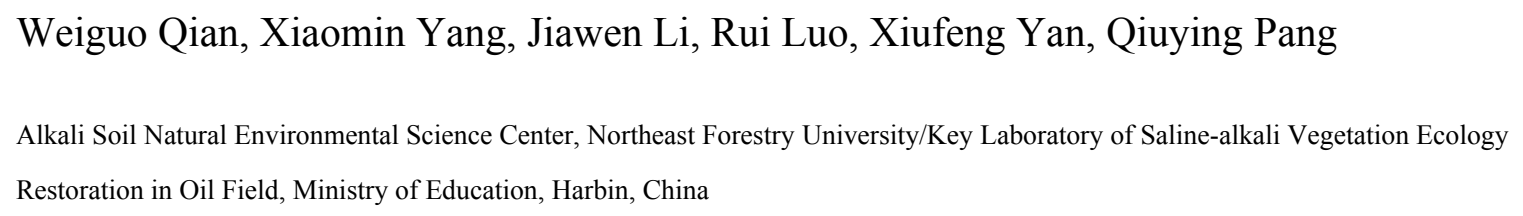

\section{Abstract}

Aquaporins (AQPs) serve as water channel proteins and belong to major intrinsic proteins (MIPs) family, functioning in rapidly and selectively transporting water and other small solutes across biological membranes. Importantly, AQPs have been shown to play a critical role in abiotic stress response pathways of plants. As a species closely related to Arabidopsis thaliana, Eutrema salsugineum has been proposed as a model for studying salt resistance in plants. Here we surveyed 35 full-length $A Q P$ genes in E. salsugineum, which could be grouped into four subfamilies including 12 plasma membrane intrinsic proteins (PIPs), 11 tonoplast intrinsic proteins (TIPs), 9 NOD-like intrinsic proteins (NIPs), and 3 small basic intrinsic proteins (SIPs) by phylogenetic analysis. EsAQPs were comprised of 237-323 amino acids, with a theoretical molecular weight (MW) of 24.31-31.80 kDa and an isoelectric point (pI) value of 4.73-10.49. Functional prediction based on the NPA motif, aromatic/arginine (ar/R) selectivity filter, Froger's position and specificity-determining position suggested quite differences in substrate specificities of EsAQPs. EsAQPs exhibited global expressions in all organs as shown by gene expression profiles and should be play important roles in response to salt, cold and drought stresses. This study provides comprehensive bioinformation on AQPs in E. salsugineum, which would be helpful for gene function analysis for further studies. 
34

35

36

37

38

39

40

41

42

43

44

45

46

47

48

49

50

51

52

53

54

55

56

57

58

59

60

61

62

63

64

65

66

67

68

69

70

71

72

73

\section{Introduction}

Water is the most abundant molecule in living cells, forming the basic medium in which all biochemical reactions take place (Dev and Herbert, 2018). Aquaporins (AQPs) belong to the major intrinsic proteins (MIPs) superfamily, which could selectively transport water molecules across the cell membrane. In addition, AQPs can also transport many small molecules, such as glycerol, urea, carbon dioxide $\left(\mathrm{CO}_{2}\right)$, silicon, boron, ammonia $\left(\mathrm{NH}_{3}\right)$ and hydrogen peroxide $\left(\mathrm{H}_{2} \mathrm{O}_{2}\right.$ ) (Biela et al., 1999; Gerbeau et al., 1999; Uehlein et al., 2003; Ma et al., 2006; Takano et al., 2006; Loque et al., 2005; Dynowski et al., 2008). AQP was firstly discovered in animals and subsequently found in almost all living organisms (Gomes et al., 2009). Compare to animals, plants have more robust and diverse AQPs. For instance, there are 35 AQPs in Arabidopsis thaliana, 33 in Oryza sativa, 40 in Sorghum bicolor, 72 in Glycine max, 47 in Cicer arietinum and 45 in Manihot esculenta (Johanson et al., 2001; Sakurai et al., 2005; Kadam et al., 2017; Zhang et al., 2013; Deokar et al., 2013; Putpeerawit et al., 2017).

Plant AQPs can be divided into seven subfamilies based on the protein sequence similarity analysis. Plasma membrane intrinsic proteins (PIPs) are the largest subfamily of plant AQPs. The most of the PIPs are commonly localized in the plasma membrane and are further divided into two phylogenetic groups PIP1 and PIP2. Tonoplast intrinsic proteins (TIPs) subfamily is usually localized in the tonoplast, which contain five classes TIP1, TIP2, TIP3, TIP4 and TIP5. NOD26-like intrinsic proteins (NIPs) named from NIP protein (Nodulin-26, GmNOD26), were discovered in the plasma membrane of soybean cells (Fortin et al., 1987). Small basic intrinsic proteins (SIPs) are typically localized in the endoplasmic reticulum. X intrinsic proteins (XIPs) are present in some dicots but absent in Brassicaceae and monocots (Maurel et al., 2015). GlpFlike intrinsic proteins (GIPs) are found in moss (Physcomitrella patens) and similar to bacterial glycerol channels (Danielson and Johanson, 2008; Gustavsson et al., 2005). Hybrid intrinsic proteins (HIPs) are found in fern (Selaginella moellendorffii) and moss (Physcomitrella patens, Anderberg et al., 2012; Gustavsson et al., 2005). Therefore, some classes (such as XIPs, HIPs, or GIPs) are considered to be lost during the evolution of certain plant lineages due to function redundancies (Maurel et al., 2015).

AQPs are highly conserved in molecular structure, consisting of six transmembrane $\alpha$-helical domains (TM1-TM6) linked by five loops (A-E), with both the $\mathrm{N}$ and $\mathrm{C}$ terminal having a cytoplasmic orientation. There are two highly conserved NPA (Asn-Pro-Ala) motifs in two half helices (HB and HE) of loopB and loopE at the center of the pore that have substrate selectivity (Tajkhorshid et al., 2002). The narrow aromatic/arginine (ar/R) selectivity filter is formed with four residues from TM helix 2 (H2), TM helix 5 (H5), and loop E (LE1 and LE2), which has been shown to provide a size barrier for solute permeability (Bansal and Sankararamakrishnan, 2007). Froger's position consists of five residues (P1-P5) that could transport two different types of molecules, water and glycerol (Froger et al., 1998). Moreover, it has been predicted that AQPs have nine specificity-determining positions (SDPs) for non-aqua substrates, such as ammonia, boron, carbon dioxide, hydrogen peroxide, silicon and urea, for each unique group (Hove and Bhave, 2011). 
74 Salt cress previously named as Thellungiella halophila or Thellungiella salsuginea, recently was

75

76

77

78

79

80

81

82

83

84

85

86

87

88

89

90

91

92

93

94

95

96

97

98

99

100

101

102

103

104

105

106

107

108

109

110

111 corrected to Eutrema salsugineum based on taxonomy and systematics, which is a relative close to A. thaliana (Koch and German, 2013). As a salt-sensitive plant, Arabidopsis has certain limits to study the mechanism of salt and drought resistance. In contrast, E. salsugineum, with a small genome, is quite tolerant to salt, drought and low temperature stresses, being considered to be a halophyte model plant for investigating the mechanism of plant resistance to stress ( $\mathrm{Zhu}, 2001$; Inan et al., 2004). The E. salsugineum AQPs like TsTIP1;2, TsMIP6 and TsPIP1;1 have been found to play an important role in plant response to abiotic stress (Wang et al., 2014; Sun et al., 2015; Li et al., 2018). The E. salsugineum genome was sequenced in 2012 and 2013 at the chromosome level and scaffold level, respectively (Wu et al., 2012; Yang et al., 2013), promoting the bioinformatics analysis of whole aquaporin family.

In this study, a genome-wide analysis of $A Q P$ genes was carried out in E. salsugineum, a total of 35 full-length $A Q P$ genes were identified. Based on the phylogenetic analysis, we found that the identified EsAQPs were quite similar to AtAQPs. The EsAQPs could be grouped into four subfamilies, including PIPs, TIPs, NIPs and SIPs. Protein sequences, chromosome distributions, gene structures and putative functions were analyzed for each of these members. The expression level of $E S A Q P$ genes in different organs and the abundance change of $E s A Q P$ genes in response to salt, drought and cold stresses were also investigated.

\section{Materials \& Methods}

\section{Identification and chromosomal location of EsAQPs}

The whole genome of E. salsugineum was downloaded from NCBI (https://www.ncbi.nlm.nih.gov/genome/12266, Wu et al., 2012; Yang et al., 2013). To identify E. salsugineum $A Q P$ candidate genes, a Hidden Markov Model (HMM) analysis was used. HMM profile of MIP (PF00230) was downloaded from Pfam protein family database (http://pfam.sanger.ac.uk/) and used as the query $(\mathrm{P}<0.05)$ to search for AQP proteins in the $E$. salsugineum genome. To avoid missing potential AQP members, the NCBI BLAST tool was used to search E. salaugineum AQPs and known Arabidopsis AQP protein sequences as a query, and the top five aligned sequences were considered as candidates. After removing all of the redundant sequences, the sequences of putative $E S A Q P$ genes were loaded on relative chromosomes of E. salsugineum using the SnapGene tool. The map of chromosome position of each $E s A Q P$ genes was drawn by MapInspect 1.0.

\section{Classification, phylogenetic analysis and structural features}

Multiple sequence alignments of putative AQP proteins were performed by ClustalW, and a phylogenetic tree was constructed using neighbor joining with MEGA 6.0 (Tamura et al., 2013). The transmembrane regions were detected using TOPCONS (http://topcons.cbr.su.se/pred/) and TMHMM (http://www.cbs.dtu.dk/services/TMHMM/). Protein subcellular localization of $E$. salsugineum AQPs was predicted in Plant-mPLoc (http://www.csbio.sjtu.edu.cn/bioinf/plant- 
$112 \mathrm{multi} /$ ) and WoLF PSORT (http:/www.genscript.com/wolf-psort.html). Functional predictions, 113 such as NPA motifs, ar/R filters (H2, H5, LE1 and LE2), Froger's positions (P1-P5) and nine 114 specificity-determining positions (SDP1-SDP9), were analyzed by the alignments with function 115 known AQPs (Quigley et al., 2001; Park et al., 2010; Hove and Bhave, 2011). The gene structure 116 for each EsAQP was illustrated with the Gene Structure Display Server 2.0

117 (http://gsds.cbi.pku.edu.cn/). The conserved motifs of EsAQP proteins were analyzed by MEME 118 suite (http://meme-suite.org/).

\section{Plant materials and stress treatments}

120 E. salsugineum seeds (ecotype Shandong, China) were provided by Prof. Hui Zhang (Shandong 121 Normal University, Jinan, China). The seeds were plated on $1 / 2 \mathrm{MS}$ medium and treated at $4^{\circ} \mathrm{C}$ 122 in dark for 7 days, then cultured in plant growth chamber with illumination of $150 \mu \mathrm{mol} / \mathrm{m}^{2} / \mathrm{s}$, 123 photoperiod $16 / 8 \mathrm{~h}$ of light/darkness at $25^{\circ} \mathrm{C}$ and $60 \%$ relative humidity. After one week, transfer

124

125

126

127

128

129

130

131

132

133

134

135

136

137

138

139

140

141

142

143

144

145

146

147

148

149 the seedlings into a mixed medium with soil and vermiculite $(3: 1)$. Vernalization treatment for bolting was conducted in 4-week old seedlings at $4^{\circ} \mathrm{C}$ for 4 weeks, and moved them back to growth chamber until getting flowers. Samples of roots, stems, leaves, flowers and siliques, were collected, immediately frozen in liquid nitrogen and stored at $-80^{\circ} \mathrm{C}$ for further analysis.

For abiotic stress assays, the 4-week old seedlings were exposed to $300 \mathrm{mM} \mathrm{NaCl}$ for $24 \mathrm{~h}$ as salt stress condition, treated at $4{ }^{\circ} \mathrm{C}$ for $24 \mathrm{~h}$ as cold stress, and lack of irrigation until the soil moisture content was less than $20 \%$ for 7 days as drought stress. The aerial part of seedling was collected for further analysis.

\section{RNA extraction, cDNA synthesis and qRT-PCR}

The total RNA was extracted using TRIzol reagent (Takara) following the manufacturer's protocol. The quality of the RNA was determined using an ultraviolet spectrophotometer (Thermo, BioMate 3S). After removing genomic DNA contamination with DNase I, cDNA was synthesized by using the PrimeScript ${ }^{\mathrm{TM}}$ RT Reagent Kit (Takara). Three biological replicates of cDNA samples were used for qRT-PCR analysis with three technical replicates.

Primers of EsPIP genes were designed using Primer 3.0 (http://bioinfo.ut.ee/primer3-0.4.0/) and the reference gene was taken from Wang et al (2014). All of primers were listed in Table S1. The qRT-PCR analysis was conducted in Applied Biosystems 7500 Real-Time PCR System (ABI, USA) by using SYBR Premix Ex TaqTM II (Takara). Reaction system contained $10 \mu \mathrm{l}$ SYBR Premix Ex Taq II, $2 \mu 1$ 5-fold diluted cDNA, $0.8 \mu \mathrm{l}$ of each primer $(10 \mathrm{mM})$, and $\mathrm{ddH}_{2} \mathrm{O}$ to a final volume of $20 \mu \mathrm{l}$. The PCR program was set as follows: $95^{\circ} \mathrm{C}$ for $30 \mathrm{~s}$, followed by 40 cycles of $95^{\circ} \mathrm{C}$ for $5 \mathrm{~s}$ and $60^{\circ} \mathrm{C}$ for $34 \mathrm{~s}$. Then, a melting curve was generated to analyze the specificity of each primer with a temperature shift from 60 to $95{ }^{\circ} \mathrm{C}$. The fold changes of the ESAQP genes expression under abiotic stresses were calculated with the $2^{-\Delta \Delta} \mathrm{Ct}$ method, while the gene expressions level of EsAQP genes in each organ were calculated with the ${ }^{\Delta} \mathrm{Ct}$ method. The heat map of gene expression pattern was visualized using HemI software.

\section{Subcellular localization of EsPIP1;2 and EsPIP2;1 proteins}


150 The coding sequences of EsPIP1;2 and EsPIP2;1 were amplified using primers containing the

151

152

153

154

155

156

157

158

159

160

161

162

163

164

165

166

167

168

169

170

171

172

173

174

175

176

177

178

179

180

181

182

183

184

185

186

187

$\mathrm{XbaI} / \mathrm{SalI}$ restriction site (Table S2). The purified products were subcloned into reconstructed pBI121 vector which composed of XbaI/SalI site and GFP. pBI121-EsPIP1;2-GFP and pBI121EsPIP2;1-GFP vectors were transformed into $A$. tumefaciens strain GV3101. And then transient transformation in onion epidermis according to the method of Xu et al (2014). Images of epidermal cells were taken by fluorescence microscope with a mirror unit (U-BW).

\section{Xenopus oocyte expression and osmotic water permeability assay}

The coding regions of EsPIP1;2 and EsPIP2;1 were subcloned into pCS107 vector using the restriction sites BamHI and EcoRI (see primers in Table S2). After linearization, the cRNAs were synthesized in vitro using the Sp6 mMessage mMachine kit (Ambion). Oocyte preparation, injection, and expression were performed as described by $\mathrm{Hu}$ et al (2012) with a little modification. $10 \mathrm{nl}$ water or cRNAs of EsPIP1;2 and EsPIP2;1 $(1 \mathrm{ng} / \mathrm{nl})$ were injected into oocytes, respectively. and then the oocytes were incubated at $18{ }^{\circ} \mathrm{C}$ for $48 \mathrm{~h}$ in Oocyte Culture Medium (OCM, 50\% L-15, 40\% HEPES (pH 7.4), 10\% calf serum, $0.5 \%$ penicillin and 10 $\mathrm{mg} / \mathrm{ml}$ streptomycin).The osmotic water permeability coefficient of oocytes was determined as described by Zhang and Verkman (1991). To measure the osmotic water permeability coefficient, oocytes were transferred to 5 -fold diluted OCM solution. Changes in the oocytes volume were monitored at room temperature with a microscope video system. Oocytes volumes (Vs) were calculated from the measured area of each oocyte. The osmotic Pf was calculated for the Erst 10 min using the formula $\mathrm{Pf}=\mathrm{V}_{0}\left[\mathrm{~d}\left(\mathrm{~V} / \mathrm{V}_{0}\right) / \mathrm{dt}\right] /\left[\mathrm{S}_{0} \times \mathrm{Vw}(\right.$ Osmin - Osmout $\left.)\right] . \mathrm{V}_{0}$ and $\mathrm{S}_{0}$ are the initial volume and surface area of each individual oocyte, respectively; $d\left(\mathrm{~V} / \mathrm{V}_{0}\right) / \mathrm{dt}$ is the relative volume increase per unit time; $\mathrm{Vw}$ is the molar volume of water $\left(18 \mathrm{~cm}^{3} \mathrm{~mol}^{-1}\right)$; and Osmin Osmout is the osmotic gradient between the inside and outside of the oocyte.

\section{Results}

\section{Characterization, classification and chromosome localization of EsAQPs}

To extensively identify AQPs in E. salsguineum, HMM profile of the MIP domain (PF00230) was used. As a result, a total of 35 putative EsAQPs were identified for further analysis (Table 1). To classify the AQP members, a phylogenetic tree was constructed according to the similarity of AQP protein sequences in E. salsugineum and Arabidopsis through the neighbor-joining method (Fig. 1). Based on the phylogenetic analysis, we found that the identified EsAQPs have very high similarity with AtAQPs which can be grouped into four subfamilies, including 12 PIPs, 11 TIPs, 9 NIPs and 3 SIPs. In addition, the EsPIP subfamily was further divided into two classes (5 EsPIP1s and 7EsPIP2s), the EsTIP subfamily into five classes (3 EsTIP1s, 4 EsTIP2s, 2 EsTIP3s, 1 EsTIP4s and 1 EsTIP5s), the EsNIP subfamily into seven classes (1 EsNIP1s, 1 EsNIP2s, 1 EsNIP3s, 3 EsNIP4s, 1 EsNIP5s, 1 EsNIP6s and 1 EsNIP7s), and the EsSIP subfamily into two classes (2 EsSIP1s and 1 EsSIP2s). The nomenclature of E. salsugineum AQPs was based on their phylogenetic relationship with AtAQPs (Fig. 1). These results were also supported by the existing annotation for E. salsugineum obtained from Phytozyme 
188 (https://phytozome.jgi.doe.gov/pz/portal.html\#!bulk?org=Org Esalsugineum), most of our

189

190

191

192

193

194

195

196

197

198

199

200

201

202

203

204

205

206

207

208

209

210

211

212

213

214

215

216

217

218

219

220

221

222

223

224

225

226

identified EsAQPs were matched to the existing annotations (Table S3). In addition, we have updated some annotations i.e., Thhalv $10008397 \mathrm{~m}$ and Thhalv10025910m, which were both annotated as PIP1;4 in Phytozyme, were renamed with EsPIP1;3 and EsPIP1;5, respectively, and other details were listed in Table S3. Compare to AtAQPs, PIP2;8 and NIP1;1 were missing in $E$. salsugineum. And TIP2;4 and NIP4;3 were identified in E.salsugineum, but not found in Arabidopsis, which were shared high similarity with their homologous genes. In Arabidopsis, 35 $A Q P$ genes were unevenly distributed on the five chromosomes (Feng et al., 2018). As shown in Table 1 and Figure 2, the chromosomal locations of 34 EsAQP genes were randomly assigned to all the seven chromosomes. However, chromosomal location of EsTIP2;2 could not be determined. Overall, AQPs from E. salsugineum had a very close relationship with those from Arabidopsis.

\section{Gene structure and subcellular localization analysis of EsAQPs}

Gene structure analysis of the $35 E s A Q P$ genes was performed on the Gene Structure Display Server of NCBI. Based on their mRNA and genomic DNA sequences, we found exon lengths were mostly conserved in each subfamily of EsAQP gene with same exon number, but introns varied in both length and position (Fig. 3). All members of EsPIP subfamily contained four exons with similar length (289-328, 296, 141 and 93-126 bp, respectively) and conserved sequences in the $2^{\text {nd }}$ and $3^{\text {rd }}$ exon, except for EsPIP2;4, which have a shorter $2^{\text {nd }}$ and longer $3^{\text {rd }}$ exon $(307,151,286$, and $111 \mathrm{bp})$. The majority members of EsTIP subfamily contained three exons with similar lengths, and the other members had two exons with similar lengths, except for EsTIP1;3, which had only one exon without intron. In the EsNIP subfamily, some members exhibited five exons with similar lengths, while others had four exons with varied lengths. All EsSIP subfamily genes displayed three exons with similar lengths. This description of exonintron structure provides additional evidence to support the classification results (Kong et al., 2017).

The prediction of subcellular localization showed diverse results, not always in agreement with experimentally determined localizations (reviewed in Katsuhara et al., 2008). In summary, the prediction of EsAQP subcellular localization in Plant-mPLoc showed that EsPIP, EsNIP and EsSIP subfamilies were localized in plasma membrane, while EsTIP subfamily members were localized in tonoplast membrane. Among them, EsPIP1;2 and EsTIP5;1 were localized in both tonoplast membrane and plasma membrane (Table 1). Moreover, WoLF PSORT predicts different location for EsAQPs and assigns values for that location (Table S4). The highest values list in Table 1 showed that EsPIPs were predicted to localize in plasma membrane, which were consistent with the Plant-mPLoc prediction and many other reports (Cui et al., 2008; Hu et al., 2012; Xu et al., 2014). Majority of other AQP members were predicted to localize in plasma membrane or tonoplast membrane, except for EsTIP5;1, EsNIP7;1 and EsSIP2;1, which were predicted to be associated with chloroplast, cytosol and endoplasmic reticulum, respectively. Moreover, some members showed multiple type of localization, for example, EsTIP3;1 was

Peer) reviewing PDF | (2019:02:34786:3:0:NEW 1 Aug 2019) 
227 predicted to be associated with chloroplast/cytosol/tonoplast membrane and EsTIP3;2 with 228 chloroplast/mitochondria /tonoplast membrane. The subcellular localization of most published 229 AQP homologous was consistent with the predicted results in E. salsugineum (Table 1). These 230 observations demonstrated that the subcellular localization of AQPs may be complex and diverse.

231

232

233

234

235

236

237

238

239

240

241

242

243

244

245

246

247

248

249

250

251

252

253

254

255

256

257

258

259

260

261

262

263

264

265

To verify the predictions, genes of EsPIP1;2 and EsPIP2;1 were cloned into the pBI121-GFP vector to create the $35 \mathrm{~S}$ ::EsPIP-GFP fusion proteins. The plasmid was transformed into onion epidermis by agrobacterium-mediated transformation. As shown in Fig. 4, the GFP fluorescence mainly exhibit in plasma membrane, indicated that EsPIP1;2 and EsPIP2;1 proteins were consistent with the predictions. Although not conclusive, the predicted localization could serve as a useful reference for further studies on EsAQPs protein functions in plants.

\section{Structure characteristics of EsAQPs}

Sequence analysis showed that all EsAQPs contain six transmembrane domains (TMDs) comprising 237-323 amino acids, had theoretical molecular weights (MW) of 24.31-31.80 kDa and isoelectric point (pI) values of 4.73-10.49 (Table 2). The EsPIP subfamily had a similar molecular weight of approximately $30.84 \mathrm{kDa}$. Most members of the EsNIP subfamily exhibited a similar molecular weight and isoelectric point of EsPIP subfamily. The EsTIP and EsSIP subfamilies had lower MW among the EsAQPs, and the isoelectric points of these two subfamilies were acidic and alkaline, respectively (Fig. S1).

NPA motifs, ar/R selectivity filters and Froger's positions of AQP protein sequences play critical role in channel selectivity. The sequence alignment between AtAQPs and GhAQPs was carried out to analyze the conserved domains (Quigley et al., 2001; Park et al., 2010). The results in Table 2 showed that all EsPIP subfamily members had two typical NPA motifs in loop B and loop E, with a water transport ar/R filter with amino acid of F-H-T-R. Froger's position consists of Q-S-A-F-W in most cases, except for EsPIP2;7, which had an M at the P1 position. All EsTIP subfamily had two typical NPA motifs. The ar/R was composed of H-I-A-V in EsTIP1s, H-I-G$\mathrm{R}$ in EsTIP2s and H-T/M/I-A-R in other EsTIP members, while in EsTIP5;1, it was composed of N-V-G-C. Froger's position consists of T-A/S-A-Y-W, except for EsTIP5;1 and EsTIP3;2, which had a $\mathrm{V}$ at the $\mathrm{P} 1$ position and a $\mathrm{T}$ at the $\mathrm{P} 2$ position respectively. Most members of EsNIP subfamily had two typical NPA motifs, not in EsNIP2;1 (with an NPG in LE), EsNIP5;1 and EsNIP7;1 (with an NPS in LB). The ar/R filter consists of residues like W/A-V/I-A/G-R, and Froger's position consists of F-S-A-Y-L, except for EsNIP7;1, which had a Y at the P1 position, and for EsNIP5;1 and EsNIP6; 1 had a $\mathrm{T}$ at the P2 position. The EsSIP subfamily showed a variable site in the first NPA, the alanine (A) was replaced by threonine (T), cysteine (C) or leucine (L). The ar/R filter was also inconsistent with each other: I-V-P-I in EsSIP1;1, V-F-P-I in EsSIP1;2 and S-H-G-A in EsSIP2;1. The Forger's position was composed of I-A-A-Y-W in EsSIP1s, while it was F-V-A-Y-W in EsSIP2;1.

MEME (Multiple EM for Motif Elicitation) is one of the most widely used tools for searching for novel "signals" in sets of biological sequences, include the discovery of new transcription factor

Peer) reviewing PDF | (2019:02:34786:3:0:NEW 1 Aug 2019) 
266 binding sites and protein domains (Bailey et al., 2006). Conserved motifs of EsAQP proteins 267 were predicted by MEME suite (Fig. 5). The result showed that motif 1, 2, 3, 4, 7, 8, and 10 were 268 same in all EsPIPs, and motif 2, 4, 7, and 10 were unique. In addition, motif 9 was unique in 269 EsPIP1s and can be used to distinguish EsPIP1s from EsPIP2s. This pattern of conserved motifs

270

271

272

273

274

275

276

277

278

279

280

281

282

283

284

285

286

287

288

289

290

291

292

293

294

295

296

297

298

299

300

301

302

303

304 in the PIP subfamily also occurs in other plants and PIP1s contain one unique motif (Tao et al., 2014; Yuan et al., 2017). In the EsTIP subfamily, almost all EsTIPs had two motif 1, two motif 3 , one motif 5 and one motif 6 . Except for EsTIP1;3, which had no motif 6. Motif 5 could be an identifier of EsTIPs among the AQPs of E. salsugineum except for EsTIP5;1. Most of members in NIP subfamily had two motif 1, two motif 3, and two motif 6, except for EsNIP2;1 (lose one motif 1), EsNIP3;1 (lose one motif 6) and EsNIP5;1 (lose one motif 3). The two motif 6 might be used to distinguish EsNIPs with other EsAQPs. All EsSIP subfamily carried motif 3. Motif 8 appeared in EsSIP1s but not in EsSIP2;1, so it might be an specific trait of this group. This is a common phenomenon in plant SIP subfamily contains less motifs (Tao et al., 2014; Reddy et al., 2015; Yuan et al., 2017; Kong et al., 2017). Based on these analysis, it was evident that there were structural differences in various EsAQP subfamilies, but conserved in their own subfamily.

\section{Expression pattern of EsAQPs}

The expression of $E s A Q P$ genes in different organs, including root, stem, leaf, flower and silique, was analyzed by RT-qPCR. The results showed that $35 E s A Q P$ genes were detected in all the organs (Fig. 6A). Almost all EsPIP genes were highly expressed in all organs, except for EsPIP2;5 in leaf. In addition, the EsPIP genes, EsTIP1;1, EsTIP1;2, EsNIP1;2, EsNIP5;1, ESSIP1;1 and EsSIP2;1 were also highly expressed in all organs. Some EsAQP genes, such as EsTIP2;3, EsTIP2;4, EsNIP2;1 and EsNIP3;1, were specifically highly expressed in root. Two EsTIPS (EsTIP2;2 and EsTIP5;1), three EsNIPS (EsNIP4;1, EsNIP4;3 and EsNIP7;1) and EsSIP 1;2 were highly expressed only in flower. Two EsTIPs (EsTIP3;1 and EsTIP3;2) were expressed in silique with relative high abundance. Compared analysis of each $E s A Q P$ gene between different organs revealed that most EsAQP genes showed higher expression level in flower than in other organs.

Abiotic stresses are the main limiting factors for plants during environmental conditions that induce osmotic stress and disturb water balance. AQPs play major roles in maintaining water homeostasis and responding to environmental stresses in plants. Therefore, we further investigated the expression patterns of EsAQP genes under salt, drought and cold stress by qRTPCR. The results showed that most of the $E S A Q P$ genes were up-regulated under salt and cold stress but down-regulated under drought stress (Fig. 6B). We found that five $E s A Q P$ genes were up-regulated under all the types of abiotic stresses, including EsPIP2;4, EsTIP1;2, EsNIP4;3, ESNIP5;1 and EsSIP1;2, while three ESAQP genes were down-regulated under all the types of abiotic stresses, including ESPIP1;5, EsTIP2;2 and EsTIP2;4. In addition, ESPIP1;1 and EsPIP 2;2 were specifically up-regulated under salt stress, and EsPIP2;1, EsTIP2;1, EsTIP5;1, EsNIP4;1 and EsNIP6; 1 were up-regulated only under cold stress.

\section{Water permeability of EsPIP1;2 and EsPIP2;1}

PeerJ reviewing PDF | (2019:02:34786:3:0:NEW 1 Aug 2019) 
305 Previously, AtPIP2;1 has been reported that is an integral membrane protein that facilitates water

306

307

308

309

310

311

312

313

314

315

316

317

318

319

320

321

322

323

324

325

326

327

328

329

330

331

332

333

334

335

336

337

338

339

340

341

342

transport across plasma membrane while AtPIP1;2 has no function (Li et al., 2011; Heckwolf et al., 2011). To determine the water channel activity of EsPIP1;2 and EsPIP2;1, proteins were tested in the Xenopus oocyte system. After two days of cRNA or water injection, the change rate in oocyte volume (Fig.7A) and the osmotic water permeability coefficient (Pf) (Fig. 7B) were calculated. Expression of EsPIP2;1 conferred a rapid osmotically driven increase in relative volume, while expression of EsPIP1;2 enabled an increase in relative volume at a slower rate than the water-injected oocytes. Compared with water-injected control, the oocytes expressing EsPIP1;2 and EsPIP2;1 showed 1.39-fold and 2.08-fold increase in Pf, suggesting that both EsPIP1;2 and EsPIP2;1 are functional AQP with water channel activity. Meanwhile, our result is consistent with the known information that PIP2s have high efficiency water transfer activity but PIP1s have little or no increase in the Pf (Chaumont et al., 2014).

\section{Discussion}

Gene duplication is a ubiquitous event that plays an important role in biological evolution, which may also contribute to stress tolerance via gene dosage increasing, avoiding some deleterious mutations and creating the opportunity for new function emergence (Innan and Kondrashov, 2010). AQPs are abundant, diverse and widely distributed in plants and involved in regulation of plant growth and development. From algae (two in Thalassiosira pseudonana and five in Phaeodactylum tricornutum) (Armbrust et al., 2004; Bowler et al., 2008) to fern (19 in Selaginella moellendorffii) (Danielson and Johanson, 2008) and moss (23 in Physcomitrella patens) (Anderberg et al., 2012) to higher plants (35 AQPs in Arabidopsis, 33 in Oryza sativa, 72 in Glycine max) ( Johanson et al., 2001; Sakurai et al., 2005; Zhang et al., 2013), the number of AQPs has largely increased with evolution. Here, we provide a genome-wide information of AQP family of E. salsugineum.

In previous studies, it was shown that more than $95 \%$ gene families are shared in $T$. salsuginea (the former name of E. salsugineum, Koch and German, 2013) with A. thaliana (Wu et al., 2012) or more than $80 \%$ E. salsugineum genes had high homology orthologs in A. thaliana (Yang et al., 2013). The number of AQPs identified in E. salsugineum is the same as that in A. thaliana, and their protein sequences have very high similarities. No homologies of AtAQPs, PIP2;8 and NIP1;1 were not identified in E. salsugineum, while another two AQPs, TIP2;4 and NIP4;3 were found instead, which were not existed in Arabidopsis. These differences may not be directly illustrated the superiority of $E$. salsugineum in stress resistance, the functions of EsAQPs in resistance need to be further studied.

\section{Structural analysis and functional inference of EsAQPs}

Exon-intron structural divergence commonly happened in duplicate gene evolution and even in sibling paralogs; these changes occurred through the mechanisms of gain/loss, exonization/pseudoexonization and insertion/deletion (Xu et al., 2012). In common bean (Phaseolus vulgaris L.), each aquaporin subfamily are completely conserved in number, order

Peer) reviewing PDF | (2019:02:34786:3:0:NEW 1 Aug 2019) 
343 and length of exons but varies in introns (Ariani and Gepts, 2015). The MEME motifs of the 344 AQPs were conserved in all subfamilies, while a few were deleted, unique or family-specific, 345 and a previous report also found this pattern in ZmPIPs (Bari et al., 2018). In our study, the 346 exon-intron structure of EsAQP genes and the conserved MEME motifs of EsAQP protein

347

348

349

350

351

352

353

354

355

356

357

358

359

360

361

362

363

364

365

366

367

368

369

370

371

372

373

374

375

376

377

378

379

380

381

382 sequences showed some common patterns (Fig. 3 and Fig. 5). These results indicated that the gene structure and the conserved motifs of EsAQPs shown subfamily-specific, these traits may provide new evidence to support the classification.

High conservation of signature sequences or residues was shown in plant PIP proteins. In our study (Table 2), EsPIPs showed a typical NPA motif, a highly conserved ar/R selectivity filter and Froger's position of F-H-T-R and Q/M-S-A-F-W, these characteristics are correlated with water transport activity (Quigley et al., 2001). In addition to water transport, plant PIPs also could transfer carbon dioxide, hydrogen peroxide, boric acid, and urea (Gaspar et al., 2003; Bienert et al., 2014; Heckwolf et al., 2011). According to the SDP analysis proposed by Hove and Bhave (2011), all EsPIPs had $\mathrm{H}_{2} \mathrm{O}_{2}$-type and urea-type SDPs (Table 3, Fig. S2). In addition, all EsPIP1s and EsPIP2;5 had boric acid-type SDPs, and all EsPIP1s had $\mathrm{CO}_{2}$-type SDPs, including two novel types of SDP showed in EsPIP1;3 and EsPIP1;4 which have an M in place of I in SDP2, it also have been found in RcPIPs, JcPIPs and BvPIPs (Zou et al., 2015; Zou et al., 2016; Kong et al., 2017). In addition, EsPIP2;4 owned another novel $\mathrm{CO}_{2}$-type SDPs (V-I-C-AV-E-W-D-W), with E replaced by D in SDP6. These results showed the conservation of plant PIPs in the transport of urea and hydrogen peroxide (Gaspar et al., 2003; Bienert et al., 2014), and PIP1s not PIP2s are main $\mathrm{CO}_{2}$ and boric acid channels (Heckwolf et al., 2011).

Compared to PIPs, TIPs are more diverse which have a variety of selectivity filters. Two typical NPA motifs were found in all the EsTIPs, and the ar/R filters and Froger's position were conserved in the EsTIP1s and EsTIP2s classes, but different with other classes. All the EsTIPs showed urea-type SDPs, and most of them had $\mathrm{H}_{2} \mathrm{O}_{2}$-type SDPs (except for EsTIP3;1 and EsTIP5;1). EsTIP2;1 had an $\mathrm{NH}_{3}$-type SDPs, as confirmed in Arabidopsis TIP2;1 (Loque et al., 2005). EsTIP3; 1 possessed a novel $\mathrm{NH}_{3}$-type SDPs (T-L-G-T-A-S-H-P-A) with F/T replaced by $\mathrm{G}$ in SDP3. The NIP subfamily has low intrinsic water permeability and the ability to transport solutes like glycerol and ammonia (Choi and Roberts, 2007). Most EsNIPs held two typical NPA motifs, but some varied at the third residue in the first or second NPA motif. All EsNIPs had urea-type SDPs, EsNIP1;2, EsNIP3;1 and EsNIP5;1 had $\mathrm{H}_{2} \mathrm{O}_{2}$-type SDPs. EsNIP5;1, EsNIP6;1 and EsNIP7;1 had boric acid-type SDPs, which have been found in Arabidopsis (Takano et al., 2006). EsNIP1;2 possessed a novel $\mathrm{NH}_{3}$-type SDPs with a substitution of $\mathrm{G}$ for A at SDP4. In addition, EsNIP4;1 and EsNIP4;3, which both had the substitution of $\mathrm{T}$ for K/L/N/V at SDP2. EsSIPs varied in the third residue of the first NPA motif, with diverse ar/R filters and Froger's positions. However, the residues were consistent with the corresponding SIP in Arabidopsis. AtSIP1;1 and AtSIP1;2 could transport water in the ER. AtSIP2;1 might act as an ER channel for other small molecules or ions (Ishikawa et al., 2005), and their similarity in these motifs suggests that these EsSIPs may have similar function. These results indicate that the diversity of AQPs in E. salsugineum may have crucial role in response to environmental stress. 


\section{Distinct expression profiles of $\operatorname{Es} A Q P$ genes in various organs}

384

385

386

387

388

389

390

391

392

393

394

395

396

397

398

399

400

401

402

403

404

405

406

407

408

409

410

411

412

413

414

415

416

417

418

419

420

421

Previous studies have shown that many AQPs show similar expression patterns, suggesting that they may act synergistic in some organs. For instance, PIPs and TIPs are abundant in all organs in many plant species (Quigley et al., 2001; Venkatesh et al., 2013; Reuscher et al., 2013; Zou et al., 2015; Yuan et al., 2017). The qRT-PCR results showed that the transcripts of EsAQP genes could be detected in all organs, but their expression levels were diverse (Fig. 6A). Among them, the most abundant transcripts were ESPIPS and a few EsTIPS (EsTIP1;1 and EsTIP1;2), which were consistent with previous studies, especially with Arabidopsis AQP genes (Jang et al., 2004). The high expression of these $A Q P$ genes may be related to their effective water channel function that mediates water uptake in plant (Jang et al. 2004; Gomes et al. 2009). Moreover, ESTIP3;1 and EsTIP3;2 were highly expressed in silique specifically. It has been reported that seed-specific TIP3;1 and TIP3;2 play a role in maintaining seed longevity, and as target genes of $\mathrm{ABI} 3$ transcription factor which known to be involved in seed desiccation tolerance and seed longevity (Mao and Sun., 2015). It suggested that TIP3s may be involve in cellular osmoregulation and maturation of the vacuolar apparatus to support optimal water uptake and growth of the embryo during seed development and germination (Shivaraj et al., 2017). In general, the transcript level of NIP subfamily is lower than others. However, the EsNIP5; 1 was high abundant in all organs, and some of them showed organ specific. For example, EsNIP2;1 and ESNIP3; 1 were predominant expression in root, ESNIP4;1, ESNIP4; 1 and ESNIP7; 1 were predominantly expressed in flower. These may rely on their transport function of diverse substrates (Mitani-Ueno et al., 2011). Strikingly, the SIP1;1 and SIP2;1 exhibited higher expression than many TIPS and NIPs in both E. salsugineum(this study) and Arabidopsis (Alexandersson et al., 2005). Compared with different organs, many AQP genes are mainly expressed in roots and flowers, whereas no $A Q P$ isoform is leaf specific in Arabidopsis (Alexandersson et al., 2005). These results were also observed in our investigation. Above all, the parallel expression patterns of $A Q P$ genes in different organs between $E$. salsugineum and Arabidopsis may further indicated their similarity.

\section{Stress responsive $A Q P$ genes in $E$. salsugineum}

Environmental stress factors such as salt, drought and low temperature can quickly reduce water transport rates (Javot and Maurel, 2003), thus the maintenance of osmotic potential is a major challenge for plants. Since AQPs are known to be involved in the maintenance of water balance in the plant, we investigated the expression of EsAQP genes at aerial parts of seedlings under various abiotic stresses including salt, drought and cold. In Arabidopsis, most AQP genes are down-regulated upon drought stress in leaves, with the exception of AtPIP1;4 and AtPIP2;5, which are up-regulated (Alexandersson et al., 2005). Besides, the expression analysis of AtPIPS at aerial parts show that only the PIP2;5 was up-regulated by cold treatment, and most of the AtPIP genes were down-regulated by cold stress whereas less-severely modulated by high salinity (Jang et al., 2004). In our data (Fig. 6B), major AQP genes of E. salsugineum were down-regulated expression to drought treatment, however, nine genes (ESPIP2;4, EsPIP2;5, 
422

423

424

425

426

427

428

429

430

431

432

433

434

435

436

437

438

439

440

441

442

443

444

445

446

447

448

449

450

451

452

453

454

455

456

EsTIP1;2, EsTIP2;3, EsTIP3;2, EsNIP1;2, EsNIP4;3, EsNIP5;1 and EsSIP1;2) were upregulated. Among these, the level of EsTIP3;2 was most significantly increased after drought treatment, which has low abundance in leaf (Fig. 6A). It is suggested that EsTIP3;2 may play a unique role under drought stress. While most of $A Q P$ genes were up-regulated under salt stress, it is consistent with those in barley and bamboo (Hove et al., 2015; Sun et al., 2016). Contrary to Arabidopsis, most of AQP genes in E. salsugineum were up-regulated under cold stress. This type of expression pattern has been reported in Sorghum bicolor (Reddy et al., 2015), to improve water transport efficiency and enhance cold tolerance ( $L i$ et al., 2008). Moreover, EsPIP1;5 was down-regulated under abiotic stresses but highly abundant in all organs, the ESTIP1;2 and ESNIP 5; 1 were highly abundant in all organs and up-regulated under various stresses. These $A Q P$ genes were induced by external stimuli, and implied to play role in maintaining water homeostasis during environmental stress (Jang et al., 2004).

\section{Conclusions}

In our study, a genome-wide information of E. salsugineum $A Q P$ gene family was provided. 35 $E S A Q P$ s, located in 7 chromosomes, were identified and divided into four subfamilies based on phylogenetic analysis, which was also supported by the subfamily-specific gene structure and MEME motifs analysis. Furthermore, functional properties were investigated through the analysis of ar/R filters, Froger's positions and SDPs, which have potential outputs for the widely function of EsAQPs. Moreover, the expression analysis was performed by qRT-PCR, showing $A Q P$ genes were widely involved in E. salsugineum organs development and abiotic stress response, and may have the potentially important roles in E. salsugineum. Our work not only provided a full-scale bioinformation of E. salsugineum AQP genes, but also offered a positive assessment for the underlying candidate EsAQPs in abiotic stress response.

\section{Funding}

This work was supported by the National Natural Science Foundation of China (No. 31570396) and the Fundamental Research Funds for the Central Universities (2572016DA05). The funders had no role in study design, data collection and analysis, decision to publish, or preparation of the manuscript.

\section{Acknowledgements}

The authors appreciate those contributors who make the Eutrema salsugineum genome data accessible in public databases.

\section{References}

Ahamed A, Murai-Hatano M, Ishikawa-Sakurai J, Hayashi H, Kawamura Y, Uemura M. 2012. Cold stress-induced acclimation in rice is mediated by root-specific aquaporins. Plant Cell Physiology 53:1445-1456 DOI 10.1093/pcp /pcs089 
457

458

459

460

461

462

463

464

465

466

467

468

469

470

471

472

473

474

475

476

477

478

479

480

481

482

483

484

485

486

487

488

489

490

491

492

493

494

495

496

Alexandersson E, Fraysse L, Sjövall-Larsen S, Gustavsson S, Fellert M, Karlsson M, Johanson U, Kjellbom P. 2005. Whole gene family expression and drought stress regulation of aquaporins. Plant Molecular Biology 59:469-484 DOI 10.1007/s11103-005-0352-1

Alexandersson E, Jonas ÅHD, Råde J, Moparthi VK, Fontes M, Kjellbom P. Johanson U. 2010. Transcriptional regulation of aquaporins in accessions of Arabidopsis in response to drought stress. The Plant Journal 61:11 DOI 10.1111/j.1365-313X.2009.04087.x Anderberg HI, Kjellbom P, Johanson U. 2012. Annotation of Selaginella moellendorffii major intrinsic proteins and the evolution of the protein family in terrestrial plants. Frontiers in Plant Science 3:33 DOI 10.3389/fpls.2012.00033

Ariani A, Gepts P. 2015. Genome-wide identification and characterization of aquaporin gene family in common bean (Phaseolus vulgaris, L.). Molecular Genetics \& Genomics 290:17711785 DOI 10.1007/s00438-015-1038-2

Armbrust EV, Berges JA, Bowler C, Green BR, Martinez D, Putnam NH, Zhou S, Allen AE, Apt KE, Bechner M, Brzezinski MA, Chaal BK, Chiovitti A, Davis AK, Demarest MS, Detter JC, Glavina T, Goodstein D, Hadi MZ, Hellsten U, Hildebrand M, Jenkins BD, Jurka J, Kapitonov VV, Kröger N, Lau WW, Lane TW, Larimer FW, Lippmeier JC, Lucas S, Medina M, Montsant A, Obornik M, Parker MS, Palenik B, Pazour GJ, Richardson PM, Rynearson TA, Saito MA, Schwartz DC, Thamatrakoln K, Valentin K, Vardi A, Wilkerson FP, Rokhsar DS. 2004. The Genome of the diatom Thalassiosira pseudonana: ecology, evolution, and metabolism. Science 306:79-86 DOI 10.1126/science. 1101156

Bailey TL, Williams N, Misleh C, Li WW. 2006. Meme: discovering and analyzing dna and protein sequence motifs. Nucleic Acids Research 34:W369-W373 DOI 10.1093/nar/gk1198 Bansal A, Sankararamakrishnan R. 2007. Homology modeling of major intrinsic proteins in rice, maize and Arabidopsis: comparative analysis of transmembrane helix association and aromatic/arginine selectivity filters. BMC Structural Biology 7:27-27 DOI 10.1186/1472-6807-727

Bari A, Farooq M, Hussain A, Muhammad TUQ, Abbas MW, Mustafa G, Karim A, Ahmed I, Hussain T. 2018. Genome-wide bioinformatics analysis of aquaporin gene family in maize (Zea mays L.). Journal of Phylogenetics \& Evolutionary Biology 6:2 DOI 10.4172/23299002.1000197

Biela A, Grote K, Otto B, Hoth S, Hedrich R, Kaldenhoff R. 1999. The Nicotiana tabacum plasma membrane aquaporin NtAQP1 is mercury-insensitive and permeable for glycerol. Plant Journal 18:565-570 DOI 10.1046/j.1365-313X.1999.00474.x

Bienert GP, Heinen RB, Berny MC, Chaumont F. 2014. Maize plasma membrane aquaporin ZmPIP2;5, but not ZmPIP1;2, facilitates transmembrane diffusion of hydrogen peroxide.

Biochimica et Biophysica Acta 1838:216-222 DOI 10.1016/j.bbamem.2013.08.011

Bowler C, Allen AE, Badger JH, Grimwood J, Jabbari K, Kuo A, Maheswari U, Martens C, Maumus F, Otillar RP, Rayko E, Salamov A, Vandepoele K, Beszteri B, Gruber A, Heijde M, Katinka M, Mock T, Valentin K, Verret F, Berges JA, Brownlee C, Cadoret JP, 
497 Chiovitti A, Choi CJ, Coesel S, De Martino A, Detter JC, Durkin C, Falciatore A, Fournet 498 J, Haruta M, Huysman MJ, Jenkins BD, Jiroutova K, Jorgensen RE, Joubert Y, Kaplan A, 499 Kröger N, Kroth PG, La Roche J, Lindquist E, Lommer M, Martin-Jézéquel V, Lopez PJ, 500 Lucas S, Mangogna M, McGinnis K, Medlin LK, Montsant A, Oudot-Le Secq MP, Napoli 501 C, Obornik M, Parker MS, Petit JL, Porcel BM, Poulsen N, Robison M, Rychlewski L, 502 Rynearson TA, Schmutz J, Shapiro H, Siaut M, Stanley M, Sussman MR, Taylor AR, 503 Vardi A, von Dassow P, Vyverman W, Willis A, Wyrwicz LS, Rokhsar DS, Weissenbach J, 504 Armbrust EV, Green BR, Van de Peer Y, Grigoriev IV. 2008. The Phaeodactylum genome 505 reveals the evolutionary history of diatom genomes. Nature 456: 239-244 DOI 506 10.1038/nature07410

507 Chaumont F, Tyerman SD. 2014. Aquaporins: highly regulated channels controlling plant 508 water relations. Plant Physiology 164: 1600-1618 DOI 10.1104/pp.113.233791

509 Choi WG, Roberts DM. 2007. Arabidopsis NIP2;1, a major intrinsic protein transporter of 510 lactic acid induced by anoxic stress. Journal of Biological Chemistry 282:24209-24218 DOI 511 10.1074/jbc.M700982200

512 Chunhui X, Meng W, Li Z, Taiyong Q, Guangmin X, Jauhar A. 2013. Heterologous 513 expression of the wheat aquaporin gene TaTIP2;2 compromises the abiotic stress tolerance of 514 Arabidopsis thaliana. PLOS ONE 8:e79618 DOI 10.1371/journal.pone.0079618

515 Cui XH, Hao FS, Chen H, Chen J, Wang XC. 2008. Expression of the Vicia faba VfPIP1gene 516 in Arabidopsis thaliana plants improves their drought resistance. Journal of Plant Research 121: 517 207-214 DOI 10.1007/s10265-007-0130-z

518 Danielson JÅ, Johanson U. 2008. Unexpected complexity of the aquaporin gene family in the 519 moss Physcomitrella patens. BMC Plant Biology 8:45 DOI 10.1186/1471-2229-8-45

520 Deokar AA, Tar'an B. 2016. Genome-wide analysis of the aquaporin gene family in Chickpea

521 (Cicer arietinum L.). Frontiers in Plant Science 7:1802 DOI 10.3389/fpls.2016.01802

522 Dev TB, Herbert JK. 2018. From aquaporin to ecosystem: plants in the water cycle. Journal of

523 Plant Physiology 227:1-2 DOI 10.1016/j.jplph.2018.06.008

524 Di Giorgio JA, Bienert GP, Nicolás Daniel Ayub, Agustín Yaneff, Muschietti JP. 2016.

525 Pollen-specific aquaporins NIP4;1 and NIP4;2 are required for pollen development and 526 pollination in Arabidopsis thaliana. The Plant Cell 28:5 DOI 10.1105/tpc.15.00776

527 Dynowski M, Schaaf G, Loque D, Moran O, Ludewig U. 2008. Plant plasma membrane water 528 channels conduct the signaling molecule $\mathrm{H}_{2} \mathrm{O}_{2}$. Biochemical Journal 414:53-61 DOI 529 10.1042/BJ20080287

530 Feng ZJ, Xu SC, Liu N, Zhang GW, Hu QZ, Xu ZS, Gong YM. 2017. Identification of the 531 AQP members involved in abiotic stress responses from Arabidopsis. Gene 646:64-73 DOI 532 10.1016/j.gene.2017.12.048

533 Fortin MG, Morrison NA, Verma DPS. 1987. Nodulin-26, a peribacteroid membrane nodulin 534 is expressed independently of the development of the peribacteroid compartment. Nucleic Acids 535 Research 15:813-824 DOI 10.1093/nar/15.2.813 
536 Froger A, Tallur B, Thomas D, Delamarche C. 1998. Prediction of functional residues in 537 water channels and related proteins. Protein Science 7:1458-1468 DOI 10.1002/pro.5560070623

538 Gaspar M, Bousser A, Sissoëff I, Roche O, Hoarau J, Mahé A. 2003. Cloning and 539 characterization of ZmPIP1-5b, an aquaporin transporting water and urea. Plant Science 165:2154031 DOI 10.1016/j.jinsphys.2013.08.013

541 Gattolin S, Sorieul M, Frigerio L. 2011. Mapping of tonoplast intrinsic proteins in maturing 542 and germinating Arabidopsis seeds reveals dual localization of embryonic TIPs to the tonoplast 543 and plasma membrane. Molecular Plant 4:180-189 DOI:10.1093/mp/ssq051

544 Gerbeau P, Guclu J, Ripoche P, Maurel C. 1999. Aquaporin Nt-TIPa can account for the high 545 permeability of tobacco cell vacuolar membrane to small neutral solutes. Plant Journal 18:577546587 DOI 10.1046/j.1365-313x.1999.00481.x

547 Gomes D, Agasse A, Thiébaud P, Delrot S, Gerós H, Chaumont F. 2009. Aquaporins are 548 multifunctional water and solute transporters highly divergent in living organisms. Biochimica et 549 Biophysica Acta 1788:1213-1228 DOI 10.1016/j.bbamem.2009.03.009

550 Gustavsson S, Lebrun AS, Kristina N, François C, Johanson U. 2005. A novel plant major 551 intrinsic protein in Physcomitrella patens most similar to bacterial glycerol channels. Plant 552 Physiology 139:287-295 DOI 10.1104/pp.105.063198

553 Hachez C, Laloux T, Reinhardt H, Cavez D, Degand H, Grefen C, Rycke RD, Inzė D, Blatt 554 MR, Russinova E, Chaumont F. 2014. Arabidopsis SNAREs SYP61 and SYP121 coordinate 555 the trafficking of plasma membrane aquaporin PIP2;7 to modulate the cell membrane water 556 permeability. The Plant Cell 26:3132-3147 DOI 10.1105/tpc.114.127159

557 Hanaoka H, Uraguchi S, Takano J, Tanaka M, Fujiwara T. 2014. OsNIP3; 1, a rice boric

558 acid channel, regulates boron distribution and is essential for growth under boron-deficient

559 conditions. The Plant Journal 78:890-902 DOI 10.1111/tpj.12511

560

561

562 Heckwolf M, Pater D, Hanson DT, Kaldenhoff R. 2011. The Arabidopsis thaliana aquaporin AtPIP1;2 is a physiologically relevant $\mathrm{CO}_{2}$ transport facilitator. Plant Journal 67:795-804 DOI 10.1111/j. 1365-313X.2011.04634.x

563

Hove RM, Bhave M. 2011. Plant aquaporins with non-aqua functions: deciphering the signature

564 sequences. Plant Molecular Biology 75:413-430 DOI 10.1007/s11103-011-9737-5

565 Hove RM, Mark Z, Mrinal B, Ricardo A. 2015. Identification and expression analysis of the

566 barley (Hordeum vulgare L.) aquaporin gene family. PLOS ONE 10:e0128025 DOI

567 10.1371/journal.pone.0128025

568 Hu W, Yuan Q, Wang Y, Cai R, Deng X, Wang J, Zhou S, Chen M, Chen L, Huang C, Ma 569 Z, Yang G, He G. 2012. Overexpression of a wheat aquaporin gene, TaAQP8, enhances salt 570 stress tolerance in transgenic Tobacco. Plant and Cell Physiology 53:2127-2141DOI

$571 \quad 10.1093 / \mathrm{pcp} / \mathrm{pcs} 154$

572 Inan G, Zhang Q, Li P, Wang Z, Cao Z, Zhang H, Zhang C, Quist TM, Goodwin SM, Zhu 573 J, Shi H, Damsz B, Charbaji T, Gong Q, Ma S, Fredricksen M, Galbraith DW, Jenks MA, 574 Rhodes D, Hasegawa PM, Bohnert HJ, Joly RJ, Bressan RA, Zhu JK. 2004. Salt Cress. A

575 halophyte and cryophyte Arabidopsis relative model system and its applicability to molecular 
576 genetic analyses of growth and development of extremophiles. Plant Physiology 135:1718-37

577 DOI 10.1104/pp.104.041723

578 Innan H, Kondrashov F. 2010. The evolution of gene duplications: classifying and

579 distinguishing between models. Nature Reviews Genetics 11: 97-108 DOI 10.1038/nrg2689

580 Ishikawa F, Suga S, Uemura T, Sato MH, Maeshima M. 2005. Novel type aquaporin SIPs are

581 mainly localized to the er membrane and show cell-specific expression in Arabidopsis thaliana.

582 FEBS Letters 579:5814-5820 DOI 10.1016/j.febslet.2005.09.076

583 Jang JY, Kim DG, Kim YO, Kim JS, Kang H. 2004. An expression analysis of a gene family 584 encoding plasma membrane aquaporins in response to abiotic stresses in Arabidopsis thaliana.

585 Plant Molecular Biology 54:713-725 DOI 10.1023/b:plan.0000040900.61345.a6

586 Javot H, Maurel C. 2002. The role of aquaporins in root water uptake. Annals of Botany

587 90:301-313 DOI 10.1093/aob/mcf199

588 Johanson U, Karlsson M, Johansson I, Gustavsson S, Sjövall S, Fraysse L, Weig AR,

589 Kjellbom P. 2001. The complete set of genes encoding major intrinsic proteins in Arabidopsis

590 provides a framework for a new nomenclature for major intrinsic proteins in plants. Plant

591 Physiology 126:1358-1369 DOI 10.1104/pp.126.4.1358

592 Kadam S, Abril A, Dhanapal AP, Koester RP, Vermerris W, Jose S, Fritschi FB. 2017.

593 Characterization and regulation of aquaporin genes of sorghum [Sorghum bicolor (L.) Moench]

594 in response to waterlogging stress. Frontiers in Plant Science 8:862 DOI

$59510.3389 /$ fpls.2017.00862

596 Katsuhara M, Hanba YT, Shiratake K, Maeshima M. 2008. Expanding roles of plant

597 aquaporins in plasma membranes and cell organelles. Functional Plant Biology 35: 1-14 DOI

598 10.1071/FP07130

599 Koch MA, German DA. 2013. Taxonomy and systematics are key to biological information:

600 Arabidopsis, Eutrema (Thellungiella), Noccaea and Schrenkiella (Brassicaceae) as examples.

601 Frontiers in Plant Science 4:267 DOI 10.3389/fpls.2013.00267

602 Kong W, Yang S, Wang Y, Bendahmane M, Fu X. 2017. Genome-wide identification and

603 characterization of aquaporin gene family in Beta vulgaris. Peer J 5:e3747 DOI

$604 \quad 10.7717 /$ peerj.3747

605 Leitäo L, Prista C, Moura TF, Loureiro-Dias MC, Soveral G. 2012. Grapevine aquaporins:

606 gating of a tonoplast intrinsic protein (TIP2;1) by cytosolic pH. PLOS ONE 7:e33219 DOI

607 10.1371/journal.pone.0033219

608 Li GW, Peng YH, Yu X, Zhang MH, Cai WM, Sun WN, Su WA. 2008. Transport functions

609 and expression analysis of vacuolar membrane aquaporins in response to various stresses in rice.

610 Journal of Plant Physiology 165:1879-1888 DOI 10.1016/j.jplph.2008.05.002

611 Li L, Wang H, Gago J, Cui H, Qian Z, Kodama N, Ji H, Tian S, Shen D, Chen Y, Sun F,

612 Xia Z, Ye Q, Sun W, Flexas J, Dong H. 2015. Harpin hpa1 interacts with aquaporin PIP1;4 to

613 promote the substrate transport and photosynthesis in Arabidopsis. Scientific Reports 5:17207

614 DOI 10.1038/srep17207 
615 Li W, Qiang XJ, Han XR, Jiang LL, Zhang SH, Han J, He R, Cheng XG. 2018. Ectopic

616 expression of a Thellungiella salsuginea aquaporin gene, TsPIP1;1, increased the salt tolerance

617 of Rice. International Journal of Molecular Science 19:2229 DOI 10.3390/ijms19082229

618 Li X, Wang X, Yang Y, Li R, He Q, Fang X, Luu DT, Maurel C, Lin J. 2011. Single-

619 molecule analysis of PIP2;1 dynamics and partitioning reveals, multiple modes of Arabidopsis

620 plasma membrane aquaporin regulation. The Plant Cell 23:3780-3797 DOI

$621 \quad 10.1105 /$ tpc. 111.091454

622 Liu C, Fukumoto T, Matsumoto T, Gena P, Frascaria D, Kaneko T, Katsuhara M, Zhong

623 S, Sun X, Zhu,Y, Iwasaki I, Ding X, Calamita G, Kitagawa Y. 2013. Aquaporin OsPIP1;1

624 promotes rice salt resistance and seed germination. Plant Physiology and Biochemistry 63:151-

625158 DOI 10.1016/j.plaphy.2012.11.018

626 Loquė D, Ludewig U, Yuan L, Wirèn NV. 2005. Tonoplast intrinsic proteins AtTIP2;1 and

627 AtTIP2;3 facilitate $\mathrm{NH}_{3}$ transport into the vacuole. Plant Physiology 137:671-680 DOI

628 10.1104/pp.104.051268

629 Ma JF, Tamai K, Yamaji N, Mitani N, Konishi S, Katsuhara M, Ishiguro M, Murata Y,

630 Yano M. 2006. A silicon transporter in rice. Nature 440:688-691 DOI 10.1038/nature04590

631 Ma S, Quist TM, Ulanov A, Joly R, Bohnert HJ. 2004. Loss of TIP1;1 aquaporin in

632 Arabidopsis leads to cell and plant death. The Plant Journal 40:845-859 DOI 10.1111/j.1365-

633 313X.2004.02265.x

634 Maurel C, Boursiac Y, Luu DT, Santoni V, Shahzad Z, Verdoucq L. 2015. Aquaporins in

635 plants. Physiological Reviews 95:1321-1358 DOI 10.1152/physrev.00008.2015

636 Mao Z, Sun W. 2015. Arabidopsis seed-specific vacuolar aquaporins are involved in

637 maintaining seed longevity under the control of abscisic acid insensitive 3. Journal of

638 Experimental Botany 66:4781-4794 DOI 10.1093/jxb/erv244

639 Mitani-Ueno N, Yamaji N, Zhao FJ, Ma JF. 2011. The aromatic/arginine selectivity filter of

640 NIP aquaporins plays a critical role in substrate selectivity for silicon, boron, and arsenic.

641 Journal of Experimental Botany 62:4391-4398 DOI 10.1093/jxb/err158

642 Mizutani M, Watanabe S, Nakagawa T, Maeshima M. 2006. Aquaporin NIP2;1 is mainly

643 localized to the ER membrane and shows root-specific accumulation in Arabidopsis thaliana.

644 Plant and Cell Physiology 47:1420-1426 DOI 10.1093/pcp/pc1004

645 Park W, Scheffler BE, Bauer PJ, Campbell BT. 2010. Identification of the family of

646 aquaporin genes and their expression in upland cotton (Gossypium hirsutum L.). BMC Plant

647 Biology 10:142 DOI 10.1186/1471-2229-10-142

648 Putpeerawit P, Sojikul P, Thitamadee S, Narangaiavana J. 2017. Genome-wide analysis of

649 aquaporin gene family and their responses to water-deficit stress conditions in cassava. Plant

650 Physiology and Biochemistry 121:118-127 DOI 10.1016/j. plaphy.2017.10.025

651 Quigley F, Rosenberg JM, Shacharhill Y, Bohnert HJ. 2001. From genome to function: the

652 Arabidopsis aquaporins. Genome Biology 3:1-17 DOI 10.1186/gb-2001-3-1-research0001 
653 Reddy PS, Rao TSRB, Sharma KK, Vadez V. 2015. Genome-wide identification and 654 characterization of the aquaporin gene family in Sorghum bicolor (L.). Plant Gene 1:18-28 DOI 655 10.1016/j.plgene.2014.12.002

656 Reuscher S, Akiyama M, Mori C, Aoki K, Shibata D, Shiratake K. 2013. Genome-wide 657 identification and expression analysis of aquaporins in tomato. PLOS ONE 8:e79052 DOI 658 10.1371/journal.pone.0079052

659 Sakurai J, Ishikawa F, Yamaguchi T, Uemura M, Maeshima M. 2005. Identification of 33 660 rice aquaporin genes and analysis of their expression and function. Plant Cell Physiology 661 46:1568-1577 DOI 10.1093/pcp/pci172

662 Shivaraj SM, Deshmukh RK, Rai R, Bélanger R, Agrawal PK, Dash PK. 2017. Genome663 wide identification, characterization, and expression profile of aquaporin gene family in flax 664 (Linum usitatissimum). Scientific Reports 7:46137 DOI 10.1038/srep46137

665 Soto G, Fox R, Ayub N, Alleva K, Muschietti J. 2010. TIP5;1 is an aquaporin specifically 666 targeted to pollen mitochondria and is probably involved in nitrogen remobilization in 667 Arabidopsis thaliana. The Plant Journal 64:1038-1047 DOI 10.1111/j.1365-313X.2010.04395.x

668 Sreedharan S, Shekhawat UKS, Ganapathi TR. 2013. Transgenic banana plants

669 overexpressing a native plasma membrane aquaporin MusaPIP1;2 display high tolerance levels 670 to different abiotic stresses. Plant Biotechnology Journal 11: 942-952 DOI 10.1111/pbi.12086

671 Sreedharan S, Shekhawat UKS, Ganapathi TR. 2015. Constitutive and stress-inducible 672 overexpression of a native aquaporin gene (MusaPIP2;6) in transgenic banana plants signals its 673 pivotal role in salt tolerance. Plant Molecular Biology 88:41-52 DOI 10.1007/s11103-015-03056742

675 Sun HY, Li LC, Lou YF, Zhao HS, Gao ZM. 2016. Genome-wide identification and 676 characterization of aquaporin gene family in moso bamboo (Phyllostachys edulis). Molecular 677 Biology Report 43:437-450 DOI 10.1007/s11033-016-3973-3

678 Sun LL, Yu GH, Han XR, Xin SC, Qiang XJ, Jiang LL, Zhang SH, Cheng XG. 2015.

679 TsMIP6 enhances the tolerance of transgenic rice to salt stress and interacts with target proteins.

680 Journal of Plant Biology 58:285-292 DOI 10.1007/s12374-015-0069-x

681 Surbanovski N, Sargent DJ, Else MA, Simpson DW, Zhang H, Grant OM. 2013. Expression 682 of Fragaria vesca PIP aquaporins in response to drought stress: PIP down-regulation correlates 683 with the decline in substrate moisture content. PLOS ONE 8: 74945 DOI

684 10.1371/journal.pone.0074945

685 Taji T, Seki M, Satou M, Sakurai T, Kobayashi M, Kanako I, Narusaka Y, Narusaka M, 686 Tajkhorshid E, Nollert P, Jensen MØ, Miercke LJ, O'Connell J, Stroud RM, Schulten K. 687 2002. Control of the selectivity of the aquaporin water channel family by global orientational 688 tuning. Science 296:525-530 DOI 10.1126/science.1067778

689 Takano J, Wada M, Ludewig U, Schaaf G, von Wiren N, Fujiwara T. 2006. The Arabidopsis 690 major intrinsic protein NIP5;1 is essential for efficient boron uptake and plant development 691 under boron limitation. Plant Cell 18:1498-1509 DOI 10.1105/tpc.106.041640 
692 Tamura K, Stecher G, Peterson D, Filipski A, Kumar S. 2013. MEGA6: molecular

693

694

695

696

697

698

699

700

701

702

703

704

705

706

707

708

709

710

711

712

713

714

715

716

717

718

719

720

721

722

723

724

725

726

727

728

729

evolutionary genetics analysis version 6.0. Molecular Biology and Evolution 30:2725-2729 DOI 10.1093/molbev/mst197

Tanaka M, Wallace IS, Takano J, Roberts DM, Fujiwara T. 2008. NIP6; 1 is a boric acid channel for preferential transport of boron to growing shoot tissues in Arabidopsis. The Plant Cell 20:2860-2875 DOI 10.1105/tpc.108.058628

Tao P, Zhong X, Li B, Wang W, Yue Z, Lei J, Guo W, Huang X. 2014. Genome-wide identification and characterization of aquaporin genes (AQPs) in Chinese cabbage (Brassica rapa ssp. pekinensis). Molecular Genetics and Genomics 289:1131-1145 DOI 10.1007/s00438014-0874-9

Uehlein N, Lovisolo C, Siefritz F, Kaldenhoff R. 2003. The tobacco aquaporin NtAQP1 is a membrane $\mathrm{CO}_{2}$ pore with physiological functions. Nature 425:734-737 DOI 10.1038/nature02027

Venkatesh J, Yu JW, Park SW. 2013. Genome-wide analysis and expression profiling of the Solanum tuberosum aquaporins. Plant Physiology \& Biochemistry 73:392-404 DOI 10.1016/j.plaphy.2013.10.025

Wang LL, Chen AP, Zhong NQ, Liu N, Wu XM, Wang F, Yang CL, Romero MF, Xia GX. 2014. The Thellungiella salsuginea tonoplast aquaporin TsTIP1;2 functions in protection against multiple abiotic stresses. Plant \& Cell Physiology 55:148-161 DOI 10.1093/pcp/pct166

Wang Y, Li R, Li D, Jia X, Zhou D, Li J, Lyi SM, Hou S, Huang Y, Kochian LV, Liu J. 2017. NIP1;2 is a plasma membrane-localized transporter mediating aluminum uptake, translocation, and tolerance in Arabidopsis. PNAS 114:5047 DOI 10.1073/pnas.1618557114 Wu HJ, Zhang Z, Wang JY, Oh DH, Dassanayake M, Liu B, Huang Q, Sun HX, Xia R, Wu Y, Wang YN, Yang Z, Liu Y, Zhang W, Zhang H, Chu J, Yan C, Fang S, Zhang J, Wang Y, Zhang F, Wang G, Lee SY, Cheeseman JM, Yang B, Li B, Min J, Yang L, Wang J, Chu C, Chen SY, Bohnert HJ, Zhu JK, Wang XJ, Xie Q. 2012. Insights into salt tolerance from the genome of Thellungiella salsuginea. Proceedings of the National Academy of Sciences 109:12219-12224 DOI 10.1073/pnas.1209954109

Xu GX, Guo CC, Shan HY, Kong HZ. 2012. Divergence of duplicate genes in exon-intron structure. Proceedings of the National Academy of Sciences 109:1187-1192 DOI 10.1073/pnas.1109047109

Xu Y, Hu W, Liu J, Zhang J, Jia C, Miao H, Xu B, Jin Z. 2014. A banana aquaporin gene, MaPIP1;1 is involved in tolerance to drought and salt stresses. BMC Plant Biology 14: 59 DOI 10.1186/1471-2229-14-59

Yang R, Jarvis DE, Chen H, Beilstein MA, Grimwood J, Jenkins J, Shu S, Prochnik S, Xin M, Ma C, Schmutz J, Wing RA, Mitchell-Olds T, Schumaker KS, Wang X. 2013. The Reference Genome of the Halophytic Plant Eutrema salsugineum. Frontiers of Plant Science 4:46 DOI 10.3389/fpls.2013.00046

Peer) reviewing PDF | (2019:02:34786:3:0:NEW 1 Aug 2019) 
730 Yuan D, Li W, Hua YP, King GJ, Xu FS, Shi L. 2017. Genome-wide identification and

731 characterization of the aquaporin gene family and transcriptional responses to boron deficiency

732 in Brassica napus. Frontiers of Plant Science 8: 1336 DOI 10.3389/fpls.2017.01336

733 Zelazny E, Miecielica U, Borst JW, Hemminga MA, Chaumont F. 2009. An N-terminal

734 diacidic motif is required for the trafficking of maize aquaporins ZmPIP2;4 and ZmPIP2;5 to the

735 plasma membrane. The Plant journal 57:346-355 DOI 10.1111/j.1365-313x.2008.03691.x

736 Zhang DY, Ali Z, Wang CB, Xu L, Yi JX, Xu ZL, Liu XQ, He XL, Huang YH, Khan IA,

737 Trethowan RM, Ma HX. 2013. Genome-wide sequence characterization and expression

738 analysis of major intrinsic proteins in soybean (Glycine max L.). PLOS ONE 8:e56312 DOI

739 10.1371/journal.pone.0056312

740 Zhu JK. 2001. Plant salt tolerance. Trends in Plant Science 6:66-71 DOI 10.1016/S1360-

741 1385(00)01838-0

742 Zou Z, Gong J, Huang QX, Mo YY, Yang LF, Xie GS. 2015. Gene structures, evolution,

743 classification and expression profiles of the aquaporin gene family in castor bean (Ricinus

744 communis L.). PLOS ONE 10:e0141022 DOI 10.1371/journal.pone.0141022

745 Zou Z, Yang L, Gong J, Mo Y, Wang J, Cao J, An F, Xie G. 2016. Genome-wide

746 identification of Jatropha curcas aquaporin genes and the comparative analysis provides insights

747 into the gene family expansion and evolution in Hevea brasiliensis. Frontiers of Plant Science

748 7:395 DOI 10.3389/fpls.2016.00395

749 
750

751

752

753

754

755

756

757

758

759

760

761

762

763

764

765

766

767

768

769

770

771

772

773

774

775

776

777

778

779

780

781

782

783

784

785

786

787

788

789

\section{Figure captions}

Figure 1 Phylogenetic tree of AQP amino acid sequences from Eutrema salsugineum and Arabidopsis thaliana. Alignments were performed using the default parameter of ClustalW and the phylogenetic tree was constructed using Neighbor-Joining tree method with 1000 bootstrap replicates in MEGA6.0 software. Each subfamily of AQPs was well separated in different clades and represented by different colors. The solid circle represents EsAQPs and the hollow circle represents AtAQPs.

Figure 2 Chromosomal localization of the EsAQP genes. The diagram was drawn using the MapInspect software, and 34 out of 35 EsAQPs were located on 7 chromosomes (except EsTIP2;2).

Figure 3 Gene structures of the EsAQP genes. The blue rectangle, yellow rectangle and black line represent UTR, exon and intron, respectively.

Figure 4 Subcelluar localizations of EsPIP1;2 and EsPIP2;1 proteins. Onion epidermal cells transiently transformed with Empty vector (A, B), EsPIP1;2-GFP (C, D) and EsPIP2;1-GFP (E, F), respectively. The images were visualized under fluorescence microscope. A, C, E: Brightfield images; B, D, F: Green fluorescence images.

Figure 5 Conserved motif analysis in EsAQPs. The conserved motif prediction was identified using MEME motif search analysis, and the maximum number parameter was set to 10 . Different motifs were represented by different colors. (A) Conserved motifs of 35 EsAQP proteins correspond to p-values. (B) Motif consensus sequences.

Figure 6 Expression profiles of the $\boldsymbol{E s} \boldsymbol{A Q P}$ genes. (A) $E s A Q P$ genes expression in response to abiotic stress. The color scale represents the $2^{-\Delta \Delta \mathrm{Ct}}$ value normalized to untreated controls and $\log _{2}$ transformed counts, where green indicates downregulated expression and red indicates upregulated expression. (B) Expression of EsAQP genes in various organs of E. salsugineum. Color scales represent $2^{\Delta \mathrm{Ct}}$ values normalized to actin and $\log _{2}$ transformed counts, where green indicates low expression and red indicates high expression.

Figure 7 Water channel activity appraisals of EsPIP1;2 and EsPIP2;1. (A) The swelling rates of Xenopus oocytes injected with $\mathrm{H}_{2} \mathrm{O}$, or cRNA encoding EsPIP1;2 and EsPIP2;1, respectively. The rate of oocyte swelling upon immersion in hypo-osmotic medium is drawn as $\mathrm{V} / \mathrm{V}_{0}$, where $\mathrm{V}$ is the volume at a given time point and $\mathrm{V}_{0}$ is the initial volume. (B) Water permeability codfficient (Pf) of oocytes injected with cRNA encoding $\mathrm{H}_{2} \mathrm{O}$, or EsPIP1;2, or EsPIP2;1. The Pf values were calculated from the rate of oocyte swelling. Vertical bars indicate the SE. Asterisks indicate significant differences in comparison with oocytes injected with water. 
790 Statistical analysis were performed by SPSS 16.0 using one-way ANOVA and Least Significant 791 Difference (LSD) test to detect significant differences $\left({ }^{*} p<0.05,{ }^{*}{ }^{*} p<0.01\right)$. 
Figure 1

Phylogenetic tree of AQP amino acid sequences from Eutrema salsugineum and Arabidopsis thaliana.

Alignments were performed using the default parameter of ClustalW and the phylogenetic tree was constructed using Neighbor-Joining tree method with 1000 bootstrap replicates in MEGA6.0 software. Each subfamily of AQPs was well separated in different clades and represented by different colors. The solid circle represents EsAQPs and the hollow circle represents AtAQPs. 


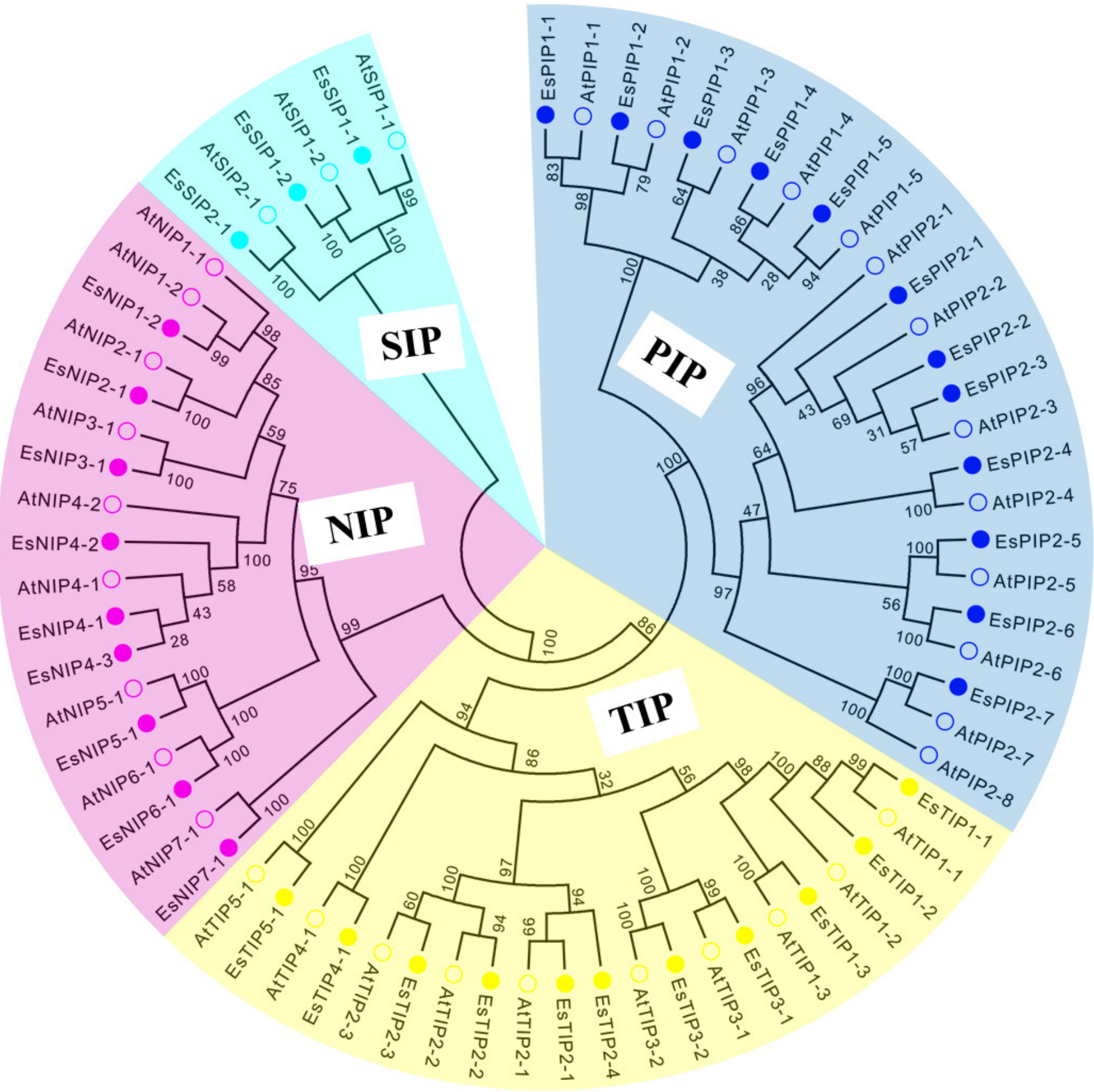


Figure 2

Chromosomal localization of the EsAQP genes.

The diagram was drawn using the Maplnspect software, and 34 out of 35 EsAQPs were located on 7 chromosomes (except EsTIP2;2).

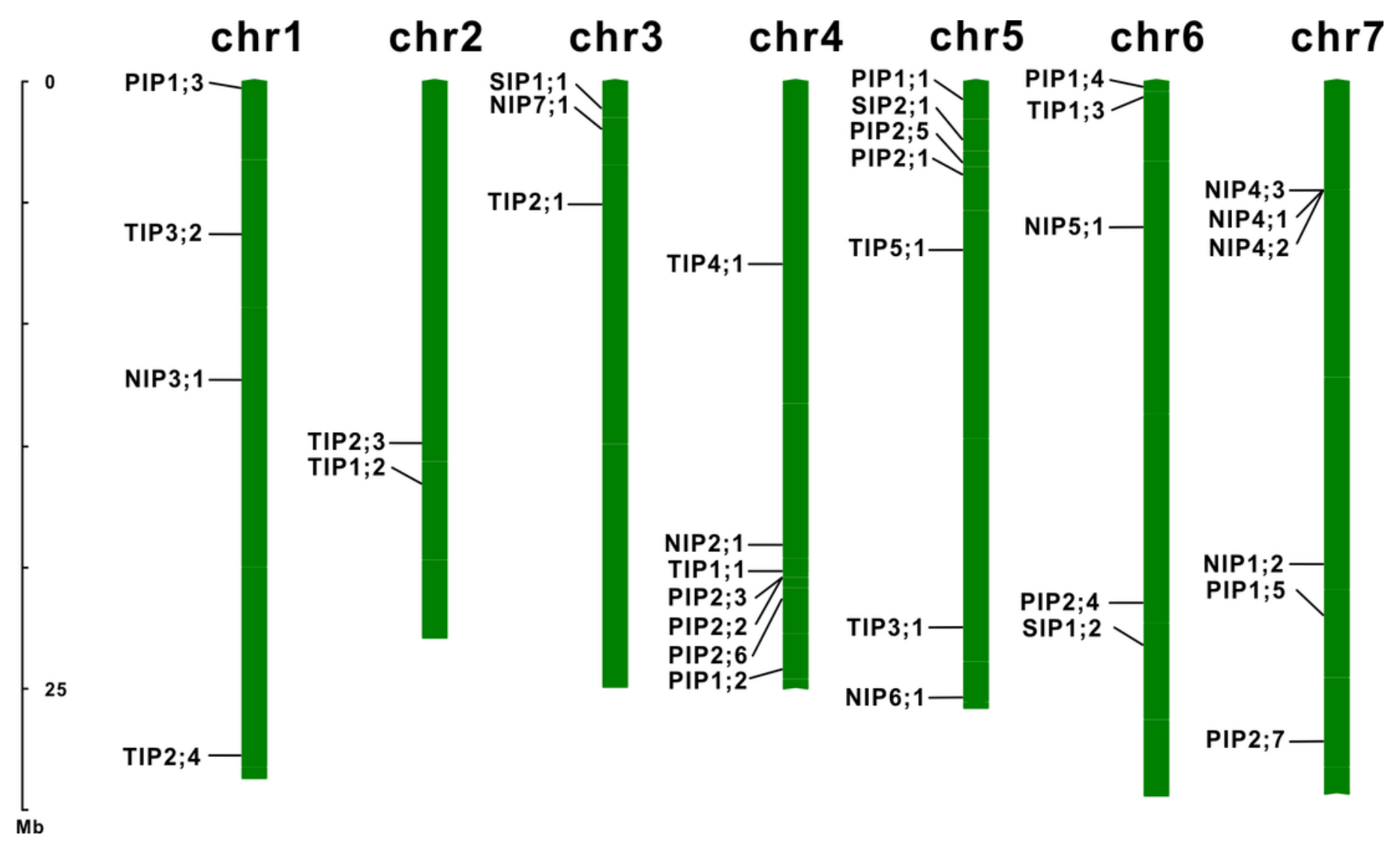


Figure 3

\section{Gene structures of the EsAQP genes.}

The blue rectangle, yellow rectangle and black line represent UTR, exon and intron, respectively.

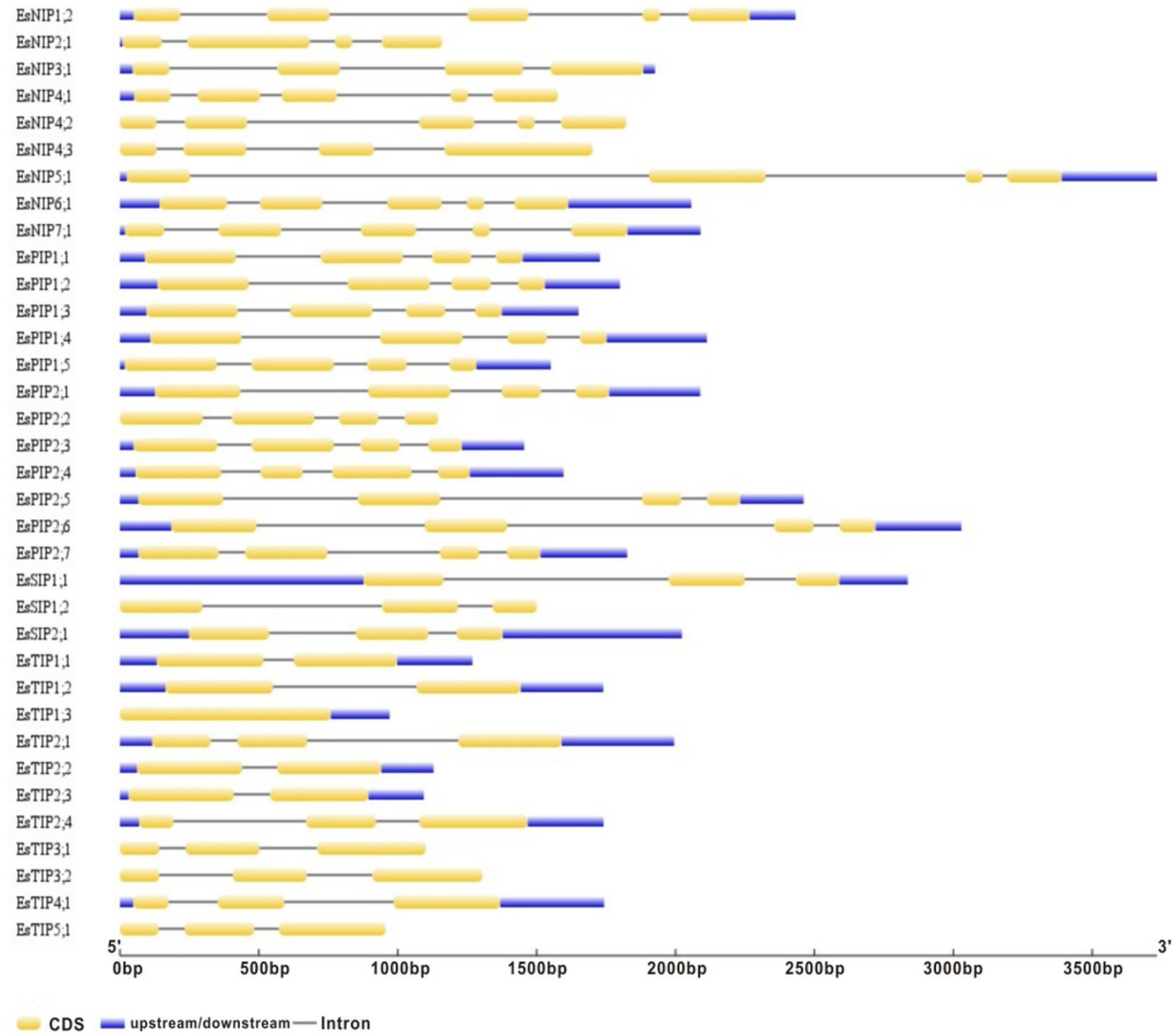




\section{Figure 4}

Subcelluar localizations of EsPIP1;2 and EsPIP2;1 proteins.

Onion epidermal cells transiently transformed with Empty vector (A, B), EsPIP1;2-GFP (C, D) and EsPIP2;1-GFP (E, F), respectively. The images were visualized under fluorescence microscope. A, C, E: Bright-field images; B, D, F: Green fluorescence images.

\section{pBI121-GFP}

pBI121-EsPIP1;2-GFP
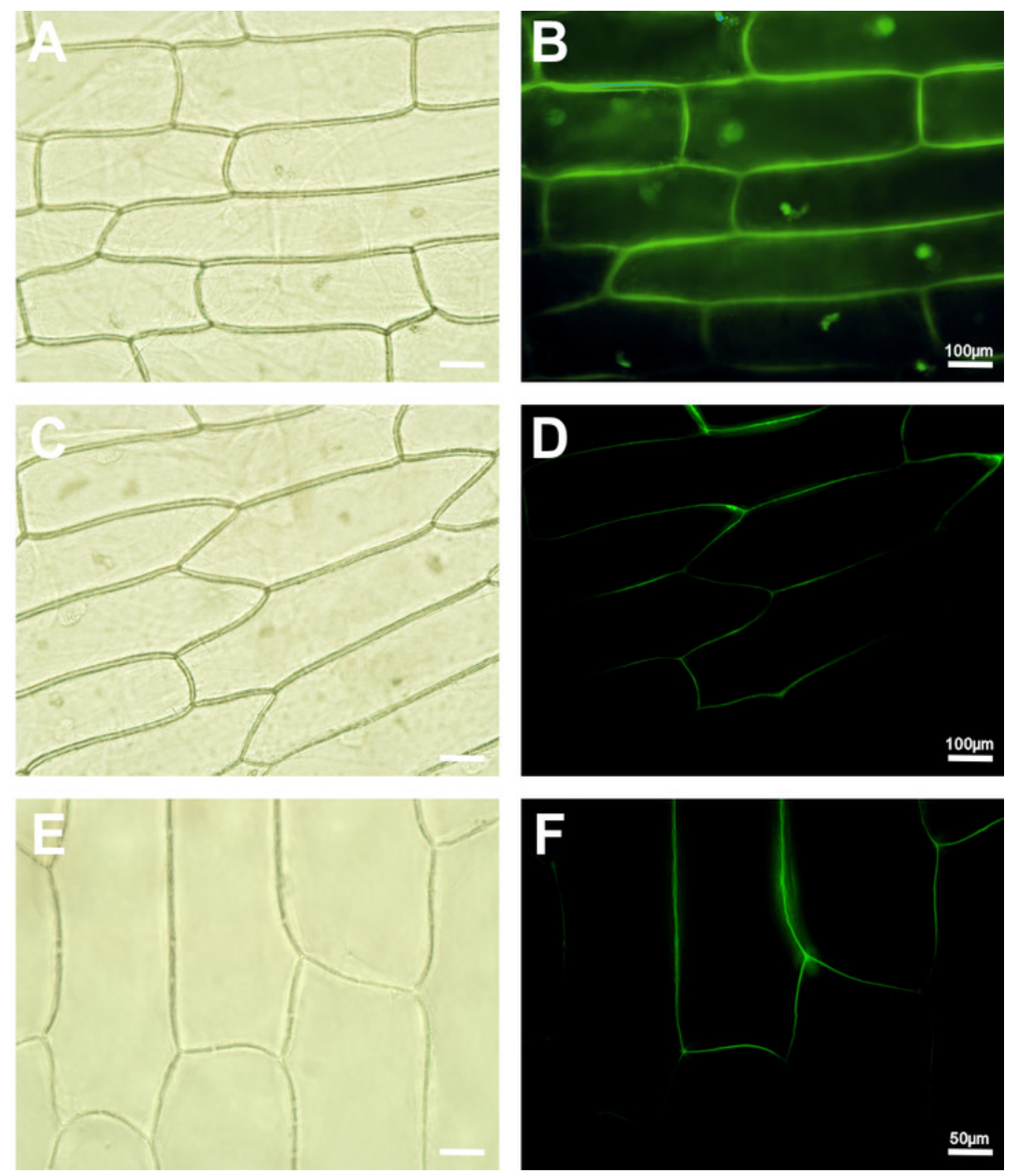


\section{Figure 5}

Conversed motif analysis in EsAQPs.

The conversed motif prediction was identified using MEME motif search analysis, and the maximum number parameter was set to 10. Different motifs were represented by different colors. (A) Conversed motifs of 35 EsAQP proteins correspond to p-values. (B) Motif consensus sequences.

A

name $p$-value

EsPIP $1: 1$ 3.916-199

EsPlP1:3 1.77 e-205

EsPP1P1; $2.880-202$

ESPIP1:5 $2.040 \cdot 20$

EsPIP2; 9.45 - 175

EsPIP2:2 9.800-174

EsPIP2: 3 2.088- 174

EsPIP2:4 6.82e-164

EsPIP2:5 4.77e-167

EsPIP2:6 $8.900-160$

EsPIP2:7 4.97e-162

EsTIP: 11 3.04e-84

EsTIP $1: 2 \quad$ 1.63e-s5

EsTIP1:3 7.59e-7

EsTIP2; $1 \quad 3.61 \mathrm{e}-90$

EstiP2:2 1.25e-s7

EsTIP2:3 $\quad$ 1.1.18e-87

EsTIP2:4 7.34e-97

EsTIP3:1 6.47e-89

EsTIP3:2 1.24e-98

EsTIP4:1 7.62e-67

EstIP5:1 2.05e-34

EsNIP1:2 $2.53 \mathrm{e}-41$

EsNIP2; $\quad 5.57$ - 35

EsNIP3:1 2.65e-37

EsNIP4:1 2.11e-42

ESNIP4:2 1.17e-4

ESNIP4:3 8.430-4s

EsNIP5: 1 1.420.32

EsNIP6:1 $9.770-39$

EsNIP7; 1 3.59e.-30

EsSIP1;1 1.15 e-18

EsSIP1:2 1.87e-15

EsSIP2; $\quad 6.89$ e.

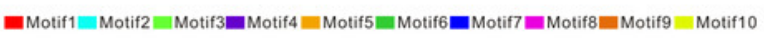

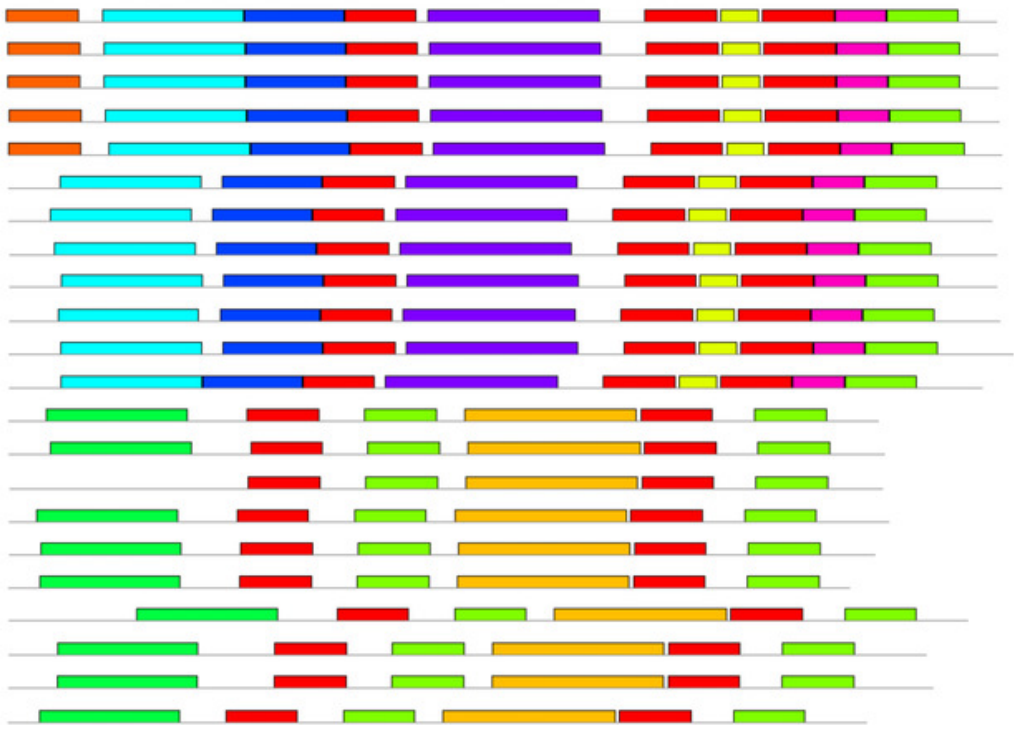

$\square \square \square$
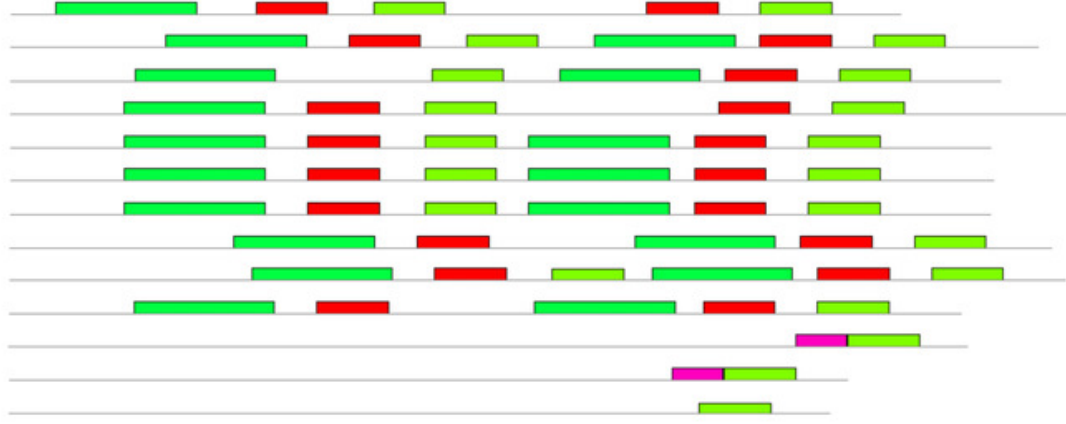

B

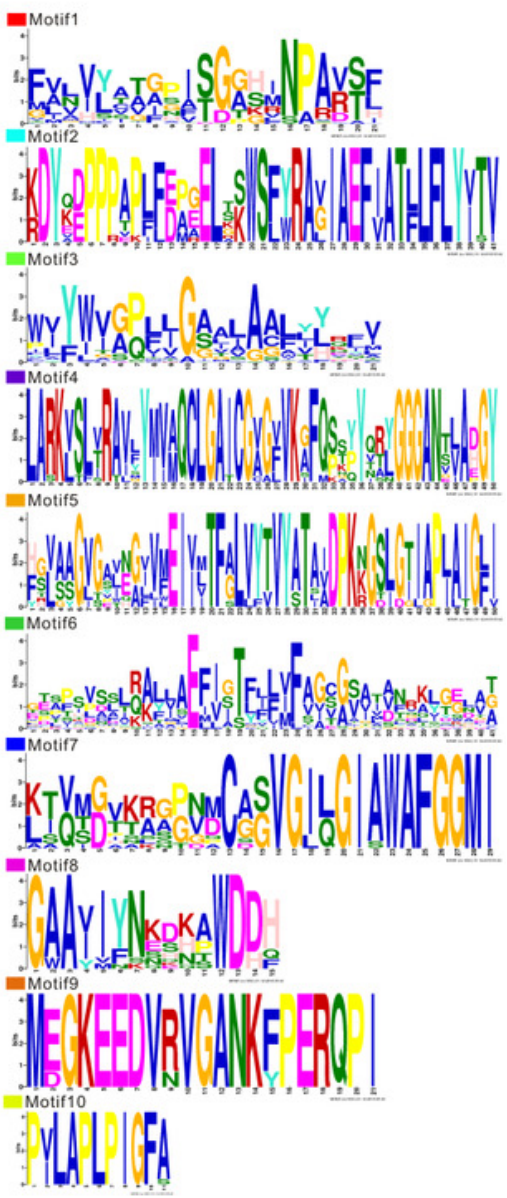




\section{Figure 6}

Expression profiles of the ESAQP genes.

(A) ESAQP genes expression in response to abiotic stress. The color scale represents the $2^{-\Delta \Delta C t}$ value normalized to untreated controls and $\log _{2}$ transformed counts, where green indicates downregulated expression and red indicates upregulated expression. (B) Expression of ESAQP genes in various organs of $E$. salsugineum. Color scales represent $2^{\Delta C t}$ values normalized to actin and $\log _{2}$ transformed counts, where green indicates low expression and red indicates high expression.

A

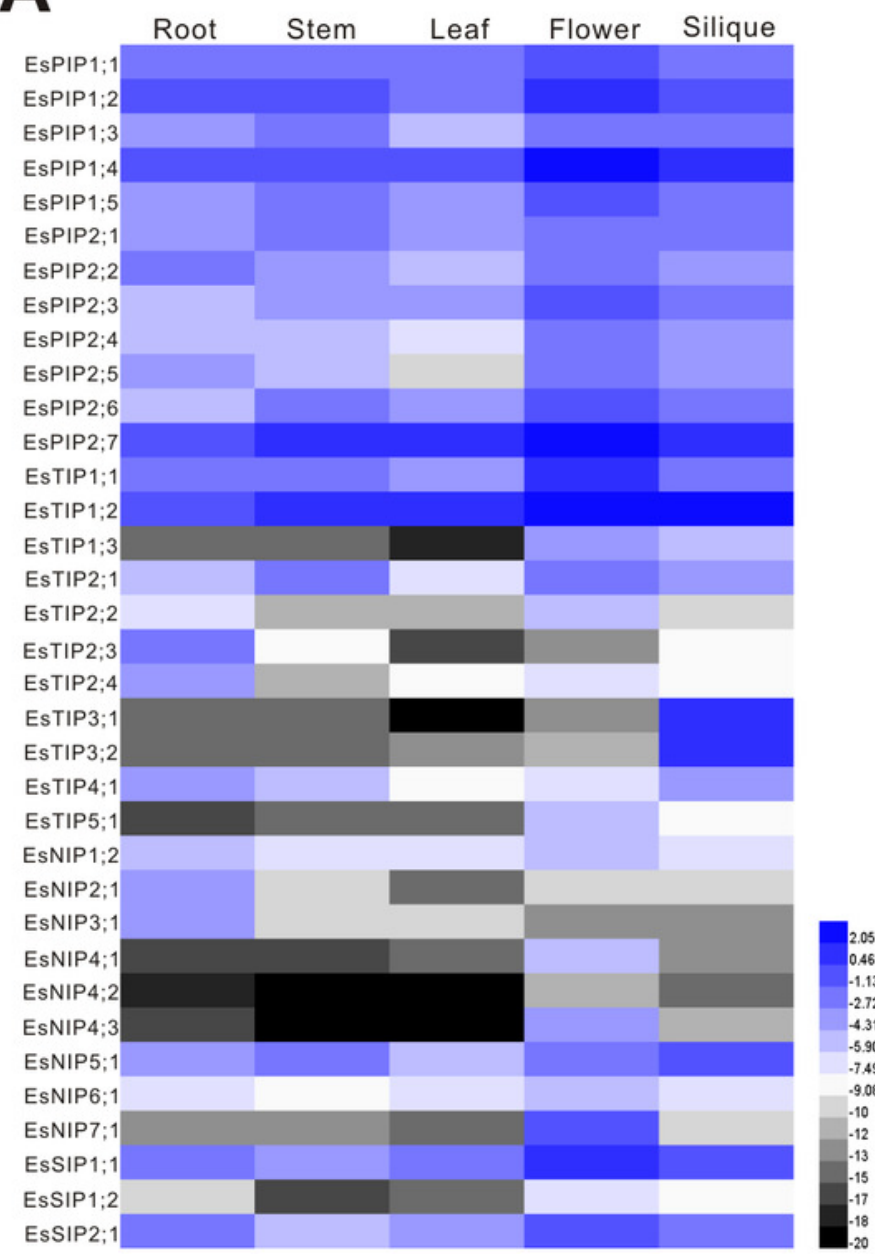

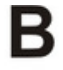

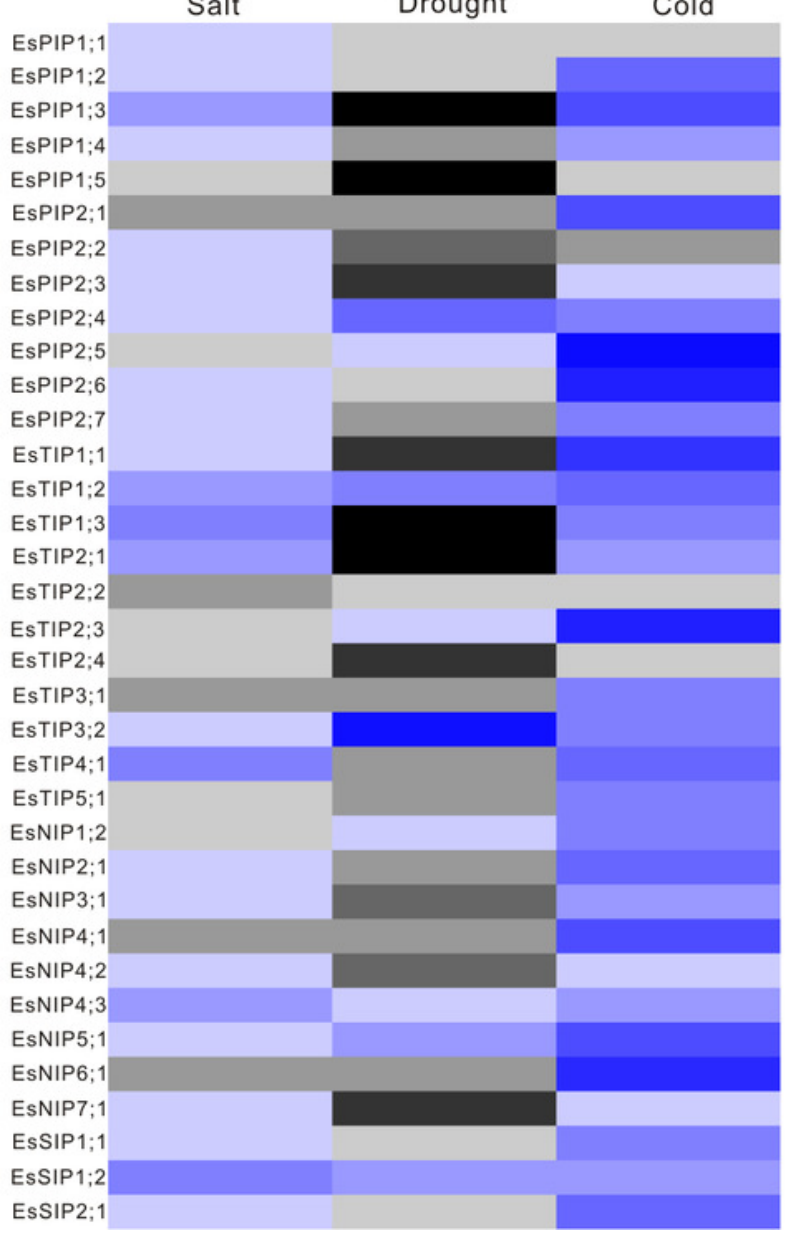




\section{Figure 7}

Water channel activity appraisals of EsPIP1;2 and EsPIP2;1.

(A) The swelling rates of Xenopus oocytes injected with $\mathrm{H}_{2} \mathrm{O}$, or cRNA encoding EsPIP1;2 and EsPIP2;1, respectively. The rate of oocyte swelling upon immersion in hypo-osmotic medium is drawn as $V / V_{0}$, where $V$ is the volume at a given time point and $V_{0}$ is the initial volume. (B) Water permeability codfficient (Pf) of oocytes injected with cRNA encoding $\mathrm{H}_{2} \mathrm{O}$, or EsPIP1;2, or EsPIP2;1. The Pf values were calculated from the rate of oocyte swelling. Vertical bars indicate the SE. Asterisks indicate significant differences in comparison with oocytes injected with water. Statistical analysis were performed by SPSS 16.0 using one-way ANOVA and Least Significant Difference (LSD) test to detect significant differences $\left({ }^{\square} p<0.05,{ }^{\square} p<0.01\right)$.
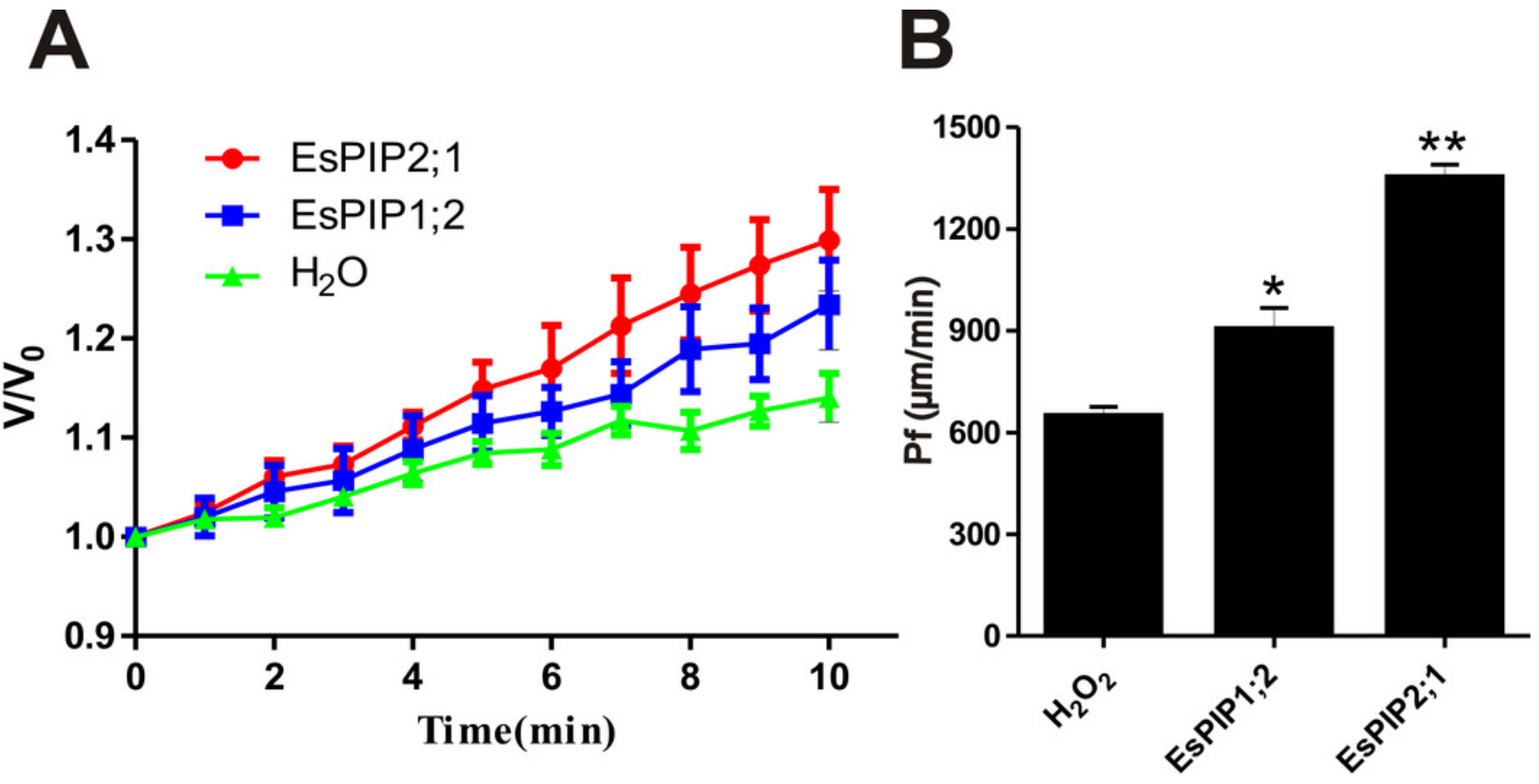


\section{Table 1 (on next page)}

Details of ESAQP genes identified from the genome-wide search analysis. 
1 TABLE1 Details of EsAQP genes identified from the genome-wide search analysis.

\begin{tabular}{|c|c|c|c|c|c|c|c|c|c|}
\hline Name & Chromosomal Localization & Scaffold & Coding sequence & Protein ID & $\begin{array}{l}\text { Plant- } \\
\text { mPLoc }\end{array}$ & WoLF PSORT & Plant species & $\begin{array}{l}\text { Subcellular } \\
\text { localization }\end{array}$ & Reference \\
\hline EsPIP1;1 & Chr5;748,014 746,287 & NW_006256838.1 & XM_006402419.1 & XP_006402482.1 & plas & plas & Oryza ativa & plas & Liu et al., 2013 \\
\hline EsPIP1;2 & Chr4;24,198,933 24,200,732 & NW_006256812.1 & XM_006397718.1 & XP_006397781.1 & plas & plas & Musa nana & plas & Sreedharan et al., 2013 \\
\hline EsPIP1;3 & Chr1;227,418 229,068 & NW_006256612.1 & XM_006418376.1 & XP_006418439.1 & plas & plas & & & \\
\hline EsPIP1;4 & Chr6;182,520 180,408 & NW_006256756.1 & XM_006396178.1 & XP_006396241.1 & plas & plas & $\begin{array}{c}\text { Arabidopsis } \\
\text { thaliana }\end{array}$ & plas & Li et al., 2015 \\
\hline EsPIP1;5 & Chr7;21,955,256 21,956,964 & NW_006256909.1 & XM_006413496.1 & XP_006413559.1 & plas & plas & & & \\
\hline EsPIP2;1 & Chr5;3,815,044 3,817,131 & NW_006256858.1 & XM_006403628.1 & XP_006403691.1 & plas & plas & A. thaliana & plas & Li et al., 2011 \\
\hline EsPIP2;2 & Chr4;20,408,518 20,407,373 & NW_006256908.1 & XM_006410833.1 & XP_006410896.1 & plas & plas & Vitis vinifera & plas & Leitäo et al., 2012 \\
\hline EsPIP2;3 & Chr4;20,411,864 20,413,318 & NW_006256908.1 & XM_006410834.1 & XP_006410897.1 & plas & plas & & & \\
\hline EsPIP2;4 & Chr6;21,418,342 21,416,629 & NW_006256829.1 & XM_006400761.1 & XP_006400824.1 & plas & plas & Zea mays & plas & Zelazny et al., 2009 \\
\hline EsPIP2;5 & Chr5;3,318,416 3,315,956 & NW_006256858.1 & XM_006403468.1 & XP_006403531.1 & plas & plas & Z. mays & plas & Zelazny et al., 2009 \\
\hline EsPIP2;6 & Chr4;21,319,556 21,322,584 & NW_006256908.1 & XM_006411061.1 & XP_006411124.1 & plas & plas & M. nana & plas & Sreedharan et al., 2015 \\
\hline EsPIP2;7 & Chr7;27,180,960 27,182,785 & NW_006256909.1 & XM_006412089.1 & XP_006412152.1 & plas & plas & A. thaliana & plas & Hachez et al., 2014 \\
\hline EsTIP1;1 & Chr4;20,182,942 20,184,210 & NW_006256908.1 & XM_006410791.1 & XP_006410854.1 & vacu & cyto & A. thaliana & vacu & Ma et al., 2004 \\
\hline EsTIP1;2 & Chr2;16,508,526 16,506,789 & NW_006256547.1 & XM_006395487.1 & XP_006395549.1 & vacu & plas/vacu & $\begin{array}{c}\text { Eutrema } \\
\text { salsiguneum }\end{array}$ & vacu & Wang et al., 2014 \\
\hline EsTIP1;3 & Chr6;663,103 662,130 & NW_006256756.1 & XM_006396285.1 & XP_006396348.1 & vacu & cyto & & & \\
\hline EsTIP2;1 & Chr3;5,624,419 5,626,413 & NW_006256885.1 & XM_006406794.1 & XP_006406857.1 & vacu & chlo/vacu & A. thaliana & vacu & Loque et al., 2005 \\
\hline EsTIP2;2 & NA & NW_006256909.1 & XM_006414179.1 & XP_006414242.1 & vacu & vacu & Triticum aestivum & vacu & Chunhui et al., 2013 \\
\hline EsTIP2;3 & Chr2;14,894,399 14,893,306 & NW_006256828.1 & XM_006398375.1 & XP_006398438.1 & vacu & vacu & A. thaliana & vacu & Loque et al., 2005 \\
\hline EsTIP2;4 & Chr1;27,709,976 27,708,236 & NW_006256486.1 & XM_006392888.1 & XP_006392950.1 & vacu & vacu & & & \\
\hline EsTIP3;1 & Chr5;22,490,388 22,491,488 & NW_006256342.1 & XM_006390520.1 & XP_006390582.1 & vacu & chlo/cyto/vacu & A. thaliana & plas/vacu & Gattolin et al., 2011 \\
\hline EsTIP3;2 & Chr1;6,309,744 6,311,048 & NW_006256612.1 & XM_006416602.1 & XP_006416665.1 & vacu & chlo/mito/vacu & A. thaliana & plas/vacu & Gattolin et al., 2011 \\
\hline EsTIP4;1 & Chr4;7,484,947 7,486,691 & NW_006256895.1 & XM_006408738.1 & XP_006408801.1 & vacu & vacu & & & \\
\hline EsTIP5;1 & Chr5;6,934,814 6,933,858 & NW_006256858.1 & XM_006404316.1 & XP_006404379.1 & vacu / plas & chlo & A. thaliana & mito & Soto et al., 2010 \\
\hline
\end{tabular}




\begin{tabular}{|c|c|c|c|c|c|c|c|c|c|}
\hline EsNIP1;2 & Chr7;19,890,089 19,892,520 & NW_006256909.1 & XM_006413978.1 & XP_006414041.1 & plas & plas & A. thaliana & plas & Wang et al., 2017 \\
\hline EsNIP2;1 & Chr4;19,043,681 19,042,522 & NW_006256908.1 & XM_006410521.1 & XP_006410584.1 & plas & vacu: & A. thaliana & plas/E.R & $\begin{array}{l}\text { Choi and Roberts, 2007; } \\
\text { Mizutani et al., } 2006\end{array}$ \\
\hline EsNIP3;1 & Chr1;12,292,410 12,294,335 & NW_006256612.1 & XM_006415218.1 & XP_006415281.1 & plas & vacu & O. sativa & plas & Hanaoka et al., 2014 \\
\hline EsNIP4;1 & $\mathrm{Chr} 7 ; 4,484,562 \sim 4,482,986$ & NW_006256877.1 & XM_006405767.1 & XP_006405830.1 & plas & plas & A. thaliana & plas/vacu & Di Giorgio et al., 2016 \\
\hline EsNIP4;2 & Chr7;4,513,301 4,511,485 & NW_006256877.1 & XM_006405768.1 & XP_006405831.1 & plas & plas & A. thaliana & plas/vacu & Di Giorgio et al., 2016 \\
\hline EsNIP4;3 & Chr7;4,481,446 4,479,745 & NW_006256877.1 & XM_006405766.1 & XP_006405829.1 & plas & plas & & & \\
\hline EsNIP5;1 & Chr6;6,005,178 6,008,910 & NW_006256756.1 & XM_006397006.1 & XP_006397069.1 & plas & plas & A. thaliana & plas & Takano et al., 2006 \\
\hline EsNIP6;1 & Chr5;25,383,958 25,386,014 & NW_006256342.1 & XM_006389768.1 & XP_006389830.1 & plas & plas & A. thaliana & plas & Tanaka et al., 2008 \\
\hline EsNIP7;1 & Chr3;1,929,290 1,927,201 & NW_006256885.1 & XM_006407920.1 & XP_006407983.1 & plas & cyto & & & \\
\hline EsSIP1;1 & Chr3;1,105,251 1,102,416 & NW_006256885.1 & XM_024159977.1 & XP_024015745.1 & plas & plas & A. thaliana & E.R & Ishikawa et al., 2005 \\
\hline EsSIP1;2 & Chr6;23,161,081 23,162,581 & NW_006256829.1 & XM_006400314.1 & XP_006400377.1 & vacu plas & vacu & A. thaliana & E.R & Ishikawa et al., 2005 \\
\hline EsSIP2; 1 & Chr5;2,401,441 2,403,463 & NW_006256838.1 & XM_006402867.1 & XP_006402930.1 & plas & E.R & A. thaliana & E.R & Ishikawa et al., 2005 \\
\hline
\end{tabular}

2 Abbreviation: plas, plasma membrane; cyto, cytosol; vacu, tonoplast membrane; chlo, chloroplast; mito, mitochondria; E.R, endoplasmic reticulum; NA,

3 Not applicable. 
Table 2 (on next page)

Structural characteristics of the EsAQPs. 
1TABLE 2 Structural characteristics of the EsAQPs.

\begin{tabular}{|c|c|c|c|c|c|c|c|c|c|c|c|c|c|c|c|}
\hline \multirow{2}{*}{ Name } & \multirow{2}{*}{ AA } & \multirow{2}{*}{$\mathrm{TM}$} & \multirow{2}{*}{$\begin{array}{l}\text { MW } \\
\text { (KD) }\end{array}$} & \multirow{2}{*}{$\mathrm{pI}$} & \multicolumn{2}{|c|}{ NPA motif } & \multicolumn{4}{|c|}{ ar/R selectivity filter } & \multicolumn{5}{|c|}{ Froger's positions } \\
\hline & & & & & LB & LE & $\mathrm{H} 2$ & H5 & LE1 & LE2 & $\mathrm{P} 1$ & $\mathrm{P} 2$ & P3 & P4 & P5 \\
\hline \multicolumn{16}{|l|}{ PIPs } \\
\hline EsPIP1;1 & 286 & 6 & 30.77 & 9.14 & NPA & NPA & $\mathrm{F}$ & $\mathrm{H}$ & $\mathrm{T}$ & $\mathrm{R}$ & Q & $\mathrm{S}$ & A & $\mathrm{F}$ & W \\
\hline EsPIP1;2 & 286 & 6 & 30.60 & 9.16 & NPA & NPA & $\mathrm{F}$ & $\mathrm{H}$ & $\mathrm{T}$ & $\mathrm{R}$ & Q & $\mathrm{S}$ & A & $\mathrm{F}$ & W \\
\hline EsPIP1;3 & 286 & 6 & 30.62 & 9.02 & NPA & NPA & $\mathrm{F}$ & $\mathrm{H}$ & $\mathrm{T}$ & $\mathrm{R}$ & Q & $\mathrm{S}$ & A & $\mathrm{F}$ & $\mathrm{W}$ \\
\hline EsPIP1;4 & 286 & 6 & 30.56 & 9.02 & NPA & NPA & $\mathrm{F}$ & $\mathrm{H}$ & $\mathrm{T}$ & $\mathrm{R}$ & Q & $\mathrm{S}$ & A & $\mathrm{F}$ & W \\
\hline EsPIP1;5 & 287 & 6 & 30.61 & 9.00 & NPA & NPA & $\mathrm{F}$ & $\mathrm{H}$ & $\mathrm{T}$ & $\mathrm{R}$ & Q & $\mathrm{S}$ & A & $\mathrm{F}$ & W \\
\hline EsPIP2;1 & 287 & 6 & 30.48 & 6.95 & NPA & NPA & $\mathrm{F}$ & $\mathrm{H}$ & $\mathrm{T}$ & $\mathrm{R}$ & $\mathrm{Q}$ & $\mathrm{S}$ & A & $\mathrm{F}$ & W \\
\hline EsPIP2;2 & 284 & 6 & 30.21 & 6.50 & NPA & NPA & $\mathrm{F}$ & $\mathrm{H}$ & $\mathrm{T}$ & $\mathrm{R}$ & $\mathrm{Q}$ & $\mathrm{S}$ & A & $\mathrm{F}$ & W \\
\hline EsPIP2;3 & 285 & 6 & 30.31 & 6.51 & NPA & NPA & $\mathrm{F}$ & $\mathrm{H}$ & $\mathrm{T}$ & $\mathrm{R}$ & $\mathrm{Q}$ & $\mathrm{S}$ & A & $\mathrm{F}$ & W \\
\hline EsPIP2;4 & 285 & 6 & 30.12 & 7.62 & NPA & NPA & $\mathrm{F}$ & $\mathrm{H}$ & $\mathrm{T}$ & $\mathrm{R}$ & $\mathrm{Q}$ & $\mathrm{S}$ & A & $\mathrm{F}$ & W \\
\hline EsPIP2;5 & 286 & 6 & 30.57 & 8.82 & NPA & NPA & $\mathrm{F}$ & $\mathrm{H}$ & $\mathrm{T}$ & $\mathrm{R}$ & $\mathrm{Q}$ & $\mathrm{S}$ & $\mathrm{A}$ & $\mathrm{F}$ & W \\
\hline EsPIP2;6 & 290 & 6 & 31.11 & 7.69 & NPA & NPA & $\mathrm{F}$ & $\mathrm{H}$ & $\mathrm{T}$ & $\mathrm{R}$ & $\mathrm{Q}$ & $\mathrm{S}$ & $\mathrm{A}$ & $\mathrm{F}$ & W \\
\hline EsPIP2;7 & 281 & 6 & 29.82 & 9.11 & NPA & NPA & $\mathrm{F}$ & $\mathrm{H}$ & $\mathrm{T}$ & $\mathrm{R}$ & $\mathrm{M}$ & $\mathrm{S}$ & A & $\mathrm{F}$ & W \\
\hline \multicolumn{16}{|l|}{ TIPs } \\
\hline EsTIP1;1 & 251 & 6 & 25.62 & 6.03 & NPA & NPA & $\mathrm{H}$ & I & A & V & $\mathrm{T}$ & A & A & $\mathrm{Y}$ & W \\
\hline EsTIP1;2 & 253 & 6 & 25.70 & 5.32 & NPA & NPA & $\mathrm{H}$ & I & A & V & $\mathrm{T}$ & A & $\mathrm{A}$ & $\mathrm{Y}$ & W \\
\hline EsTIP1;3 & 252 & 6 & 25.85 & 5.10 & NPA & NPA & $\mathrm{H}$ & I & A & V & $\mathrm{T}$ & $\mathrm{S}$ & A & $\mathrm{Y}$ & W \\
\hline EsTIP2;1 & 277 & 6 & 28.32 & 7.80 & NPA & NPA & $\mathrm{H}$ & I & G & $\mathrm{R}$ & $\mathrm{T}$ & $\mathrm{S}$ & A & $\mathrm{Y}$ & W \\
\hline EsTIP2;2 & 250 & 6 & 25.02 & 4.87 & NPA & NPA & $\mathrm{H}$ & I & G & $\mathrm{R}$ & $\mathrm{T}$ & $\mathrm{S}$ & A & $\mathrm{Y}$ & W \\
\hline EsTIP2;3 & 243 & 6 & 24.31 & 4.73 & NPA & NPA & $\mathrm{H}$ & I & G & $\mathrm{R}$ & $\mathrm{T}$ & $\mathrm{S}$ & A & $\mathrm{Y}$ & W \\
\hline EsTIP2;4 & 254 & 6 & 25.85 & 5.43 & NPA & NPA & $\mathrm{H}$ & I & G & $\mathrm{R}$ & $\mathrm{T}$ & $\mathrm{S}$ & A & $\mathrm{Y}$ & W \\
\hline EsTIP3;1 & 265 & 6 & 27.94 & 7.17 & NPA & NPA & $\mathrm{H}$ & $\mathrm{T}$ & A & $\mathrm{R}$ & $\mathrm{T}$ & A & A & $\mathrm{Y}$ & W \\
\hline EsTIP3;2 & 267 & 6 & 28.29 & 6.58 & NPA & NPA & $\mathrm{H}$ & $\mathrm{M}$ & A & $\mathrm{R}$ & $\mathrm{T}$ & $\mathrm{T}$ & A & $\mathrm{Y}$ & W \\
\hline EsTIP4;1 & 249 & 6 & 26.16 & 5.49 & NPA & NPA & $\mathrm{H}$ & I & A & $\mathrm{R}$ & $\mathrm{T}$ & $\mathrm{S}$ & A & $\mathrm{Y}$ & W \\
\hline EsTIP5;1 & 257 & 6 & 26.70 & 7.72 & NPA & NPA & $\mathrm{N}$ & $\mathrm{V}$ & G & $\mathrm{C}$ & $\mathrm{V}$ & A & A & $\mathrm{Y}$ & W \\
\hline \multicolumn{16}{|l|}{ NIPs } \\
\hline EsNIP1;2 & 297 & 6 & 31.80 & 8.83 & NPA & NPA & W & $\mathrm{V}$ & A & $\mathrm{R}$ & $\mathrm{F}$ & $\mathrm{S}$ & A & $\mathrm{Y}$ & $\mathrm{L}$ \\
\hline EsNIP2;1 & 286 & 6 & 30.56 & 6.78 & NPA & NPG & W & $\mathrm{V}$ & A & $\mathrm{R}$ & $\mathrm{F}$ & $\mathrm{S}$ & A & $\mathrm{Y}$ & $\mathrm{L}$ \\
\hline EsNIP3;1 & 323 & 6 & 34.46 & 5.94 & NPA & NPA & W & I & A & $\mathrm{R}$ & $\mathrm{F}$ & $\mathrm{S}$ & A & $\mathrm{Y}$ & $\mathrm{L}$ \\
\hline EsNIP4;1 & 283 & 6 & 30.49 & 8.73 & NPA & NPA & W & $\mathrm{V}$ & A & $\mathrm{R}$ & $\mathrm{F}$ & $\mathrm{S}$ & A & $\mathrm{Y}$ & $\mathrm{L}$ \\
\hline EsNIP4;2 & 284 & 6 & 30.34 & 8.80 & NPA & NPA & W & $\mathrm{V}$ & A & $\mathrm{R}$ & $\mathrm{F}$ & $\mathrm{S}$ & $\mathrm{A}$ & $\mathrm{Y}$ & $\mathrm{L}$ \\
\hline EsNIP4;3 & 283 & 6 & 30.30 & 8.98 & NPA & NPA & W & $\mathrm{V}$ & A & $\mathrm{R}$ & $\mathrm{F}$ & $\mathrm{S}$ & A & $\mathrm{Y}$ & $\mathrm{L}$ \\
\hline EsNIP5;1 & 301 & 6 & 31.20 & 8.31 & NPS & NPA & A & I & G & $\mathrm{R}$ & $\mathrm{F}$ & $\mathrm{T}$ & A & $\mathrm{Y}$ & $\mathrm{L}$ \\
\hline EsNIP6;1 & 305 & 6 & 31.78 & 8.57 & NPA & NPA & A & I & A & $\mathrm{R}$ & $\mathrm{F}$ & $\mathrm{T}$ & A & $\mathrm{Y}$ & $\mathrm{L}$ \\
\hline EsNIP7;1 & 275 & 6 & 28.62 & 6.12 & NPS & NPA & A & $\mathrm{V}$ & G & $\mathrm{R}$ & $\mathrm{Y}$ & $\mathrm{S}$ & A & $\mathrm{Y}$ & $\mathrm{L}$ \\
\hline \multicolumn{16}{|l|}{ SIPs } \\
\hline EsSIP1;1 & 238 & 6 & 25.41 & 9.89 & NPT & NPA & $\mathrm{I}$ & $\mathrm{V}$ & $\mathrm{P}$ & I & I & A & A & $\mathrm{Y}$ & W \\
\hline EsSIP1;2 & 242 & 6 & 25.96 & 9.83 & NPC & NPA & $\mathrm{V}$ & $\mathrm{F}$ & $\mathrm{P}$ & I & I & A & A & $\mathrm{Y}$ & W \\
\hline
\end{tabular}




\begin{tabular}{|c|c|c|c|c|c|c|c|c|c|c|c|c|c|c|c|}
\hline EsSIP2;1 & 237 & 6 & 25.85 & 9.64 & NPL & NPA & $\mathrm{S}$ & $\mathrm{H}$ & G & A & $\mathrm{F}$ & $\mathrm{V}$ & A & Y & W \\
\hline
\end{tabular}


Table 3 (on next page)

Identified typical SDPs in EsAQPs.

The red font represent novel site. 
TABLE 3 Identified typical SDPs in EsAQPs. The red font represent novel site.

\begin{tabular}{|c|c|c|c|c|c|c|c|c|c|}
\hline \multirow{2}{*}{ Aquaporin } & \multicolumn{9}{|c|}{ Specificity-determining positions } \\
\hline & SDP1 & SDP2 & SDP3 & SDP4 & SDP5 & SDP6 & SDP7 & SDP8 & SDP9 \\
\hline Ammonia Transporters & $\mathbf{F} / \mathbf{T}$ & $\mathbf{K} / \mathbf{L} / \mathrm{N} / \mathrm{V}$ & $\mathbf{F} / \mathbf{T}$ & $\mathbf{V} / \mathbf{L} / \mathbf{T}$ & $\mathbf{A}$ & $\mathbf{D} / \mathbf{S}$ & $\mathbf{A} / \mathbf{H} / \mathbf{L}$ & $\mathbf{E} / \mathbf{P} / \mathbf{S}$ & $\mathbf{A} / \mathbf{R} / \mathbf{T}$ \\
\hline EsTIP2;1 & $\mathrm{T}$ & $\mathrm{L}$ & $\mathrm{T}$ & $\mathrm{V}$ & A & $\mathrm{S}$ & $\mathrm{H}$ & $\mathrm{P}$ & A \\
\hline EsTIP3;1 & $\mathrm{T}$ & $\mathrm{L}$ & G & $\mathrm{T}$ & A & $\mathrm{S}$ & $\mathrm{H}$ & $\mathrm{P}$ & A \\
\hline EsNIP1;2 & $\mathrm{F}$ & K & $\mathrm{F}$ & $\mathrm{T}$ & G & $\mathrm{D}$ & $\mathrm{L}$ & E & $\mathrm{T}$ \\
\hline EsNIP4;1 & $\mathrm{F}$ & $\mathrm{T}$ & $\mathrm{F}$ & $\mathrm{T}$ & A & $\mathrm{D}$ & $\mathrm{L}$ & $\mathrm{E}$ & $\mathrm{T}$ \\
\hline EsNIP4;3 & $\mathrm{F}$ & $\mathrm{T}$ & $\mathrm{F}$ & $\mathrm{T}$ & A & $\mathrm{D}$ & $\mathrm{L}$ & $\mathrm{E}$ & $\mathrm{T}$ \\
\hline Boric Acid transporter & $\mathbf{T} / \mathbf{V}$ & $\mathbf{I} / \mathbf{V}$ & $\mathrm{H} / \mathrm{I}$ & $\mathbf{P}$ & $\mathbf{E}$ & $\mathbf{I} / \mathbf{L}$ & $\mathbf{I} / \mathbf{L} / \mathbf{T}$ & $\mathbf{A} / \mathbf{T}$ & $\mathbf{A} / \mathbf{G} / \mathbf{P} / \mathbf{K}$ \\
\hline EsPIP1;1 & $\mathrm{T}$ & I & $\mathrm{H}$ & $\mathrm{P}$ & E & $\mathrm{L}$ & $\mathrm{L}$ & $\mathrm{T}$ & $P$ \\
\hline EsPIP1;2 & $\mathrm{T}$ & I & $\mathrm{H}$ & $\mathrm{P}$ & E & $\mathrm{L}$ & $\mathrm{L}$ & $\mathrm{T}$ & $\mathrm{P}$ \\
\hline EsPIP1;3 & $\mathrm{T}$ & I & $\mathrm{H}$ & $\mathrm{P}$ & $\mathrm{E}$ & $\mathrm{L}$ & $\mathrm{L}$ & $\mathrm{T}$ & $P$ \\
\hline EsPIP1;4 & $\mathrm{T}$ & I & $\mathrm{H}$ & $\mathrm{P}$ & $\mathrm{E}$ & $\mathrm{L}$ & $\mathrm{L}$ & $\mathrm{T}$ & $\mathrm{P}$ \\
\hline EsPIP1;5 & $\mathrm{T}$ & I & $\mathrm{H}$ & $\mathrm{P}$ & $\mathrm{E}$ & $\mathrm{L}$ & $\mathrm{L}$ & $\mathrm{T}$ & $\mathrm{P}$ \\
\hline EsPIP2;5 & $\mathrm{T}$ & I & $\mathrm{H}$ & $\mathrm{P}$ & E & $\mathrm{L}$ & $\mathrm{L}$ & $\mathrm{T}$ & $P$ \\
\hline EsNIP5;1 & $\mathrm{T}$ & I & $\mathrm{H}$ & $\mathrm{P}$ & E & $\mathrm{L}$ & $\mathrm{L}$ & A & $\mathrm{P}$ \\
\hline EsNIP6;1 & $\mathrm{T}$ & I & $\mathrm{H}$ & $\mathrm{P}$ & $\mathrm{E}$ & $\mathrm{L}$ & $\mathrm{L}$ & A & $\mathrm{P}$ \\
\hline EsNIP7;1 & V & I & $\mathrm{H}$ & $\mathrm{P}$ & E & $\mathrm{L}$ & $\mathrm{L}$ & $\mathrm{T}$ & $\mathrm{P}$ \\
\hline $\mathrm{CO}_{2}$ transporter & $\mathbf{I} / \mathbf{L} / \mathbf{V}$ & I & C & A & $\mathbf{I} / \mathbf{V}$ & D & $\mathbf{W}$ & D & $\mathbf{W}$ \\
\hline EsPIP1;1 & $\mathrm{L}$ & I & $\mathrm{C}$ & A & I & D & W & $\mathrm{D}$ & W \\
\hline EsPIP1;2 & V & I & $\mathrm{C}$ & A & I & $\mathrm{D}$ & $\mathrm{W}$ & $\mathrm{D}$ & W \\
\hline EsPIP1;3 & V & M & $\mathrm{C}$ & A & I & $\mathrm{D}$ & $\mathrm{W}$ & $\mathrm{D}$ & W \\
\hline EsPIP1;4 & V & M & $\mathrm{C}$ & A & I & $\mathrm{D}$ & $\mathrm{W}$ & $\mathrm{D}$ & W \\
\hline EsPIP1;5 & V & I & $\mathrm{C}$ & A & I & D & W & $\mathrm{D}$ & W \\
\hline EsPIP2;4 & V & I & $\mathrm{C}$ & A & $\mathrm{V}$ & E & $\mathrm{W}$ & D & W \\
\hline $\mathrm{H}_{2} \mathrm{O}_{2}$ transporters & $\mathbf{A} / \mathbf{S}$ & $\mathbf{A} / \mathbf{G}$ & $\mathbf{L} / \mathbf{V}$ & $\mathbf{A} / \mathbf{F} / \mathbf{L} / \mathbf{V} / \mathbf{T}$ & $\mathbf{I} / \mathbf{L} / \mathbf{V}$ & $\mathbf{H} / \mathbf{I} / \mathbf{L} / \mathbf{Q}$ & $\mathbf{F} / \mathbf{Y}$ & $\mathbf{A} / \mathbf{V}$ & $\mathbf{P}$ \\
\hline EsPIP1;1 & A & G & V & $\mathrm{F}$ & I & $\mathrm{H}$ & $\mathrm{F}$ & V & $\mathrm{P}$ \\
\hline EsPIP $1 ; 2$ & A & G & V & $\mathrm{F}$ & I & $\mathrm{H}$ & $\mathrm{F}$ & V & $\mathrm{P}$ \\
\hline EsPIP1;3 & A & G & V & $\mathrm{F}$ & I & $\mathrm{H}$ & $\mathrm{F}$ & V & $\mathrm{P}$ \\
\hline EsPIP1;4 & A & G & V & $\mathrm{F}$ & I & $\mathrm{H}$ & F & V & $\mathrm{P}$ \\
\hline EsPIP1;5 & A & G & V & F & I & $\mathrm{H}$ & $\mathrm{F}$ & V & $\mathrm{P}$ \\
\hline EsPIP2;1 & A & G & V & $\mathrm{F}$ & I & $\mathrm{H}$ & $\mathrm{F}$ & V & $\mathrm{P}$ \\
\hline EsPIP $2 ; 2$ & A & G & V & F & I & $\mathrm{H}$ & $\mathrm{F}$ & V & $\mathrm{P}$ \\
\hline EsPIP2;3 & A & G & V & $\mathrm{F}$ & I & $\mathrm{H}$ & $\mathrm{F}$ & V & $\mathrm{P}$ \\
\hline EsPIP $2 ; 4$ & A & G & V & $\mathrm{F}$ & I & Q & $\mathrm{F}$ & V & $\mathrm{P}$ \\
\hline EsPIP2;5 & A & G & V & $\mathrm{F}$ & I & $\mathrm{H}$ & $\mathrm{F}$ & V & $\mathrm{P}$ \\
\hline EsPIP2;6 & A & G & V & $\mathrm{F}$ & I & Q & $\mathrm{F}$ & V & $\mathrm{P}$ \\
\hline EsPIP2;7 & A & G & V & $\mathrm{F}$ & I & $\mathrm{H}$ & $\mathrm{F}$ & V & $\mathrm{P}$ \\
\hline EsTIP1;1 & S & A & $\mathrm{L}$ & A & I & $\mathrm{H}$ & $\mathrm{Y}$ & A & $\mathrm{P}$ \\
\hline EsTIP1;2 & $\mathrm{S}$ & A & $\mathrm{L}$ & A & I & $\mathrm{H}$ & $\mathrm{Y}$ & A & $\mathrm{P}$ \\
\hline
\end{tabular}




\begin{tabular}{|c|c|c|c|c|c|c|c|c|c|}
\hline EsTIP $1 ; 3$ & A & A & $\mathrm{L}$ & $\mathrm{S}$ & I & $\mathrm{H}$ & $\mathrm{Y}$ & $\mathrm{V}$ & $\mathrm{P}$ \\
\hline EsTIP2;1 & S & A & $\mathrm{L}$ & $\mathrm{V}$ & I & $\mathrm{H}$ & $\mathrm{Y}$ & V & $\mathrm{P}$ \\
\hline EsTIP2;2 & S & A & $\mathrm{L}$ & V & I & I & $\mathrm{Y}$ & V & $\mathrm{P}$ \\
\hline EsTIP2;3 & S & A & $\mathrm{L}$ & $\mathrm{V}$ & I & I & $\mathrm{Y}$ & V & $\mathrm{P}$ \\
\hline EsTIP3;2 & A & $\mathrm{A}$ & $\mathrm{L}$ & A & I & $\mathrm{H}$ & $\mathrm{Y}$ & V & $\mathrm{P}$ \\
\hline EsTIP4;1 & $\mathrm{S}$ & A & $\mathrm{L}$ & $\mathrm{L}$ & $\mathrm{T}$ & $\mathrm{H}$ & $\mathrm{Y}$ & V & $\mathrm{P}$ \\
\hline EsNIP1;2 & $\mathrm{S}$ & A & $\mathrm{L}$ & $\mathrm{L}$ & V & I & $\mathrm{Y}$ & V & $\mathrm{P}$ \\
\hline EsNIP3;1 & S & A & $\mathrm{L}$ & $\mathrm{V}$ & I & $\mathrm{L}$ & $\mathrm{Y}$ & $\mathrm{V}$ & $P$ \\
\hline EsNIP5;1 & S & A & $\mathrm{L}$ & $\mathrm{V}$ & $\mathrm{V}$ & $\mathrm{L}$ & $\mathrm{Y}$ & $\mathrm{V}$ & $\mathrm{P}$ \\
\hline Silicic acid transporters & $\mathrm{C} / \mathrm{S}$ & $\mathbf{F} / \mathbf{Y}$ & $\mathbf{A} / \mathbf{E} / \mathbf{L}$ & $\mathbf{H} / \mathbf{R} / \mathbf{Y}$ & G & $\mathrm{K} / \mathrm{N} / \mathrm{T}$ & $\mathbf{R}$ & $\mathbf{E} / \mathbf{S} / \mathbf{T}$ & $\mathbf{A} / \mathbf{K} / \mathbf{P} / \mathbf{T}$ \\
\hline \multicolumn{10}{|l|}{ Not found } \\
\hline Urea Transporters & $\mathbf{H}$ & $\mathbf{P}$ & F/L/L/T & $\mathbf{A} / \mathbf{C} / \mathbf{F} / \mathbf{L}$ & $\mathbf{L} / \mathbf{M}$ & $\mathbf{A} / \mathbf{G} / \mathbf{P}$ & $\mathbf{G} / \mathbf{S}$ & $\mathbf{G} / \mathbf{S}$ & $\mathbf{N}$ \\
\hline EsPIP1;1 & $\mathrm{H}$ & $\mathrm{P}$ & $\mathrm{F}$ & $\mathrm{F}$ & $\mathrm{L}$ & $\mathrm{P}$ & $\mathrm{G}$ & G & $\mathrm{N}$ \\
\hline EsPIP1;2 & $\mathrm{H}$ & $\mathrm{P}$ & $\mathrm{F}$ & $\mathrm{F}$ & $\mathrm{L}$ & $\mathrm{P}$ & $\mathrm{G}$ & $\mathrm{G}$ & $\mathrm{N}$ \\
\hline EsPIP1;3 & $\mathrm{H}$ & $\mathrm{P}$ & $\mathrm{F}$ & $\mathrm{F}$ & $\mathrm{L}$ & $\mathrm{P}$ & $\mathrm{G}$ & G & $\mathrm{N}$ \\
\hline EsPIP $1 ; 4$ & $\mathrm{H}$ & $\mathrm{P}$ & $\mathrm{F}$ & $\mathrm{F}$ & $\mathrm{L}$ & $\mathrm{P}$ & $\mathrm{G}$ & G & $\mathrm{N}$ \\
\hline EsPIP $1 ; 5$ & $\mathrm{H}$ & $\mathrm{P}$ & $\mathrm{F}$ & $\mathrm{F}$ & $\mathrm{L}$ & $\mathrm{P}$ & $\mathrm{G}$ & G & $\mathrm{N}$ \\
\hline EsPIP2;1 & $\mathrm{H}$ & $\mathrm{P}$ & $\mathrm{F}$ & $\mathrm{F}$ & $\mathrm{L}$ & $\mathrm{P}$ & $\mathrm{G}$ & G & $\mathrm{N}$ \\
\hline EsPIP2;2 & $\mathrm{H}$ & $\mathrm{P}$ & $\mathrm{F}$ & $\mathrm{F}$ & $\mathrm{L}$ & $\mathrm{P}$ & $\mathrm{G}$ & G & $\mathrm{N}$ \\
\hline EsPIP2;3 & $\mathrm{H}$ & $\mathrm{P}$ & $\mathrm{F}$ & $\mathrm{F}$ & $\mathrm{L}$ & $\mathrm{P}$ & $\mathrm{G}$ & G & $\mathrm{N}$ \\
\hline EsPIP2;4 & $\mathrm{H}$ & $\mathrm{P}$ & $\mathrm{F}$ & $\mathrm{F}$ & $\mathrm{L}$ & $\mathrm{P}$ & $\mathrm{G}$ & G & $\mathrm{N}$ \\
\hline EsPIP2;5 & $\mathrm{H}$ & $\mathrm{P}$ & $\mathrm{F}$ & $\mathrm{F}$ & $\mathrm{L}$ & $\mathrm{P}$ & $\mathrm{G}$ & G & $\mathrm{N}$ \\
\hline EsPIP2;6 & $\mathrm{H}$ & $\mathrm{P}$ & $\mathrm{F}$ & $\mathrm{F}$ & $\mathrm{L}$ & $\mathrm{P}$ & $\mathrm{G}$ & G & $\mathrm{N}$ \\
\hline EsPIP2;7 & $\mathrm{H}$ & $\mathrm{P}$ & $\mathrm{F}$ & $\mathrm{F}$ & $\mathrm{L}$ & $\mathrm{P}$ & $\mathrm{G}$ & G & $\mathrm{N}$ \\
\hline EsTIP1;1 & $\mathrm{H}$ & $\mathrm{P}$ & $\mathrm{F}$ & $\mathrm{F}$ & $\mathrm{L}$ & A & $\mathrm{G}$ & $\mathrm{S}$ & $\mathrm{N}$ \\
\hline EsTIP1;2 & $\mathrm{H}$ & $\mathrm{P}$ & $\mathrm{F}$ & $\mathrm{F}$ & $\mathrm{L}$ & A & $\mathrm{G}$ & S & $\mathrm{N}$ \\
\hline EsTIP1;3 & $\mathrm{H}$ & $\mathrm{P}$ & $\mathrm{F}$ & $\mathrm{F}$ & $\mathrm{L}$ & A & $\mathrm{G}$ & S & $\mathrm{N}$ \\
\hline EsTIP2;1 & $\mathrm{H}$ & $\mathrm{P}$ & $\mathrm{F}$ & A & $\mathrm{L}$ & $\mathrm{P}$ & $\mathrm{G}$ & S & $\mathrm{N}$ \\
\hline EsTIP2;2 & $\mathrm{H}$ & $\mathrm{P}$ & $\mathrm{L}$ & A & $\mathrm{L}$ & $\mathrm{P}$ & $\mathrm{G}$ & S & $\mathrm{N}$ \\
\hline EsTIP2;3 & $\mathrm{H}$ & $\mathrm{P}$ & $\mathrm{L}$ & A & $\mathrm{L}$ & $\mathrm{P}$ & $\mathrm{G}$ & S & $\mathrm{N}$ \\
\hline EsTIP2;4 & $\mathrm{H}$ & $\mathrm{P}$ & $\mathrm{F}$ & V & $\mathrm{L}$ & $\mathrm{P}$ & $\mathrm{G}$ & S & $\mathrm{N}$ \\
\hline EsTIP3;1 & $\mathrm{H}$ & $\mathrm{P}$ & $\mathrm{F}$ & $\mathrm{L}$ & $\mathrm{L}$ & $\mathrm{P}$ & $\mathrm{G}$ & S & $\mathrm{N}$ \\
\hline EsTIP3;2 & $\mathrm{H}$ & $\mathrm{P}$ & $\mathrm{L}$ & $\mathrm{L}$ & $\mathrm{L}$ & $\mathrm{P}$ & $\mathrm{G}$ & S & $\mathrm{N}$ \\
\hline EsTIP4;1 & $\mathrm{H}$ & $\mathrm{P}$ & I & $\mathrm{L}$ & $\mathrm{L}$ & A & $\mathrm{G}$ & S & $\mathrm{N}$ \\
\hline EsTIP5;1 & $\mathrm{H}$ & $\mathrm{P}$ & $\mathrm{F}$ & A & $\mathrm{L}$ & $\mathrm{P}$ & $\mathrm{G}$ & S & $\mathrm{N}$ \\
\hline EsNIP1;2 & $\mathrm{H}$ & $\mathrm{P}$ & I & A & $\mathrm{L}$ & $\mathrm{P}$ & $\mathrm{G}$ & S & $\mathrm{N}$ \\
\hline EsNIP2;1 & $\mathrm{H}$ & $\mathrm{P}$ & I & A & $\mathrm{L}$ & E & $\mathrm{G}$ & S & $\mathrm{N}$ \\
\hline EsNIP3;1 & $\mathrm{H}$ & $\mathrm{P}$ & I & A & $\mathrm{L}$ & $\mathrm{P}$ & $\mathrm{G}$ & S & $\mathrm{N}$ \\
\hline EsNIP4;1 & $\mathrm{H}$ & $\mathrm{P}$ & V & A & $\mathrm{L}$ & $\mathrm{P}$ & $\mathrm{G}$ & S & $\mathrm{N}$ \\
\hline EsNIP4;2 & $\mathrm{H}$ & $\mathrm{P}$ & $\mathrm{F}$ & A & $\mathrm{L}$ & $\mathrm{P}$ & $\mathrm{G}$ & S & $\mathrm{N}$ \\
\hline EsNIP4;3 & $\mathrm{H}$ & $\mathrm{P}$ & I & A & $\mathrm{L}$ & $\mathrm{P}$ & $\mathrm{G}$ & S & $\mathrm{N}$ \\
\hline
\end{tabular}




\begin{tabular}{llllllllll}
\hline EsNIP5;1 & $\mathrm{H}$ & $\mathrm{P}$ & $\mathrm{I}$ & $\mathrm{A}$ & $\mathrm{L}$ & $\mathrm{P}$ & $\mathrm{G}$ & $\mathrm{S}$ & $\mathrm{N}$ \\
EsNIP6;1 & $\mathrm{H}$ & $\mathrm{P}$ & $\mathrm{I}$ & $\mathrm{A}$ & $\mathrm{L}$ & $\mathrm{P}$ & $\mathrm{S}$ & $\mathrm{S}$ & $\mathrm{N}$ \\
EsNIP7;1 & $\mathrm{H}$ & $\mathrm{P}$ & $\mathrm{I}$ & $\mathrm{A}$ & $\mathrm{V}$ & $\mathrm{P}$ & $\mathrm{G}$ & $\mathrm{S}$ & $\mathrm{N}$ \\
\hline
\end{tabular}

2 